DESY 02-166

ZU-TH 12/02

\title{
The Reconstruction of Supersymmetric Theories at High Energy Scales
}

\author{
G.A. Blair ${ }^{a, b}$, W. Porod $^{c}$, and P.M. Zerwas ${ }^{a}$ \\ ${ }^{a}$ Deutsches Elektron-Synchrotron DESY, D-22603 Hamburg, Germany \\ ${ }^{b}$ Royal Holloway, University of London, London, UK \\ ${ }^{c}$ Inst. für Theor. Physik, Universität Zürich, CH-8057 Zürich, Switzerland
}

October 28, 2018

\begin{abstract}
The reconstruction of fundamental parameters in supersymmetric theories requires the evolution to high scales, where the characteristic regularities in mechanisms of supersymmetry breaking become manifest. We have studied a set of representative examples in this context: minimal supergravity and a left-right symmetric extension; gauge mediated supersymmetry breaking; and superstring effective field theories. Through the evolution of the parameters from the electroweak scale the regularities in different scenarios at the high scales can be unravelled if precision analyses of the supersymmetric particle sector at $e^{+} e^{-}$linear colliders are combined with analyses at the LHC.
\end{abstract}

\section{Introduction}

Extending the Standard Model to a supersymmetric theory [1, 2] is an attractive step which has provided the qualitative understanding of a diverse set of phenomena in particle physics. Supersymmetry stabilizes the gap between the Grand Unification scale / Planck scale and the electroweak scale [3]. It allows the unification of the three gauge couplings at a scale $M_{U} \simeq 2 \cdot 10^{16} \mathrm{GeV}$ in a straight forward way [4. Radiative electroweak symmetry breaking relates to the high value of the top mass [5]. Moreover, the cold dark matter component in the universe can be identified with the lightest supersymmetric particle [6]. Above all, local supersymmetry, requiring the existence of massless spin 2 fields, provides a rationale for gravity [7]. 
Supersymmetry is not an exact symmetry in Nature. Unravelling the breaking mechanism is therefore one of the central issues with this new concept. A variety of mechanisms have been proposed, based on rather different physical ideas. Among these schemes are supergravity theories [8] which have provided the framework for many phenomenological analyses. The suppression of flavour-changing neutral reactions is achieved in an automatic form within gauge mediated supersymmetry breaking [9]. Supersymmetry is broken in these scenarios in a hidden sector at high and intermediate scales, respectively, and the breaking is mediated by gravity or gauge interactions to the visible sector. The breaking, however, may not be communicated by direct action from the hidden to the visible sector. This is realized in anomaly mediated supersymmetry breaking models [10] in which supersymmetric particle masses are a consequence of the superconformal anomaly. In gaugino meditated supersymmetry breaking [11, supersymmetry is broken on a 3-brane separated from the 3-brane of the visible sector, and the breaking is communicated by gauge and Higgs superfields propagating through the 5-dimensional bulk. While in many models of supersymmetry breaking the gaugino masses are assumed to be universal at the unification scale, superstring motivated models, in which the breaking is moduli dominated, as opposed to dilaton dominated scenarios, give rise to non-universal boundary conditions at the high scale for the gauginos as well as the sfermion mass parameters [12, 13. They can be exploited to determine the parameters of the string effective field theories.

In this report we elaborate on earlier investigations of Ref. [14] in which elements of gravity and gauge mediated supersymmetry breaking have been considered in realistic experimental environments of the proton collider LHC 15] and prospective $\mathrm{TeV} e^{+} e^{-}$linear colliders [16, 17]. We extend these investigations in several directions in the present report.

In supergravity inspired models we adopt a scenario close to the Snowmass Point SPS\#1 [18]. In a second step, the previous analysis, based on the Minimal Supersymmetric Standard Model, is extended to a left-right supersymmetric $S O(10)$ model 19. The $S O(10)$ symmetry is assumed to be realized at a scale between the standard $S U(5)$ scale $M_{U} \simeq 2 \cdot 10^{16}$, derived from the unification of the gauge couplings, and the Planck scale $M_{P} \simeq 10^{19} \mathrm{GeV}$. The right-handed neutrinos are assumed heavy, with masses at intermediate scales between $O\left(10^{10}\right) \mathrm{GeV}$ and $O\left(10^{15}\right) \mathrm{GeV}$, so that the observed light neutrino masses are generated by the see-saw mechanism in a natural way [20]. A rough estimate of the intermediate scale follows from the evolution of the mass parameters to the low experimental scale if universality holds at the Grand Unification scale.

In the gauge mediated symmetry breaking scenario, the fundamental scale is expected to be in the range from $\mathrm{O}(10 \mathrm{TeV})$ to $\mathrm{O}\left(10^{6} \mathrm{TeV}\right)$. We present an update and an extension of the earlier analysis. In particular, the effective supersymmetry breaking scales, the messenger and supersymmetric mass scales, can be reconstructed at the point where the masses of the sparticles carrying the same quantum numbers become identical, the characteristic regularity of gauge mediated supersymmetry breaking. 
The anomaly mediated as well as the gaugino mediated SUSY breaking are technically equivalent to the mSUGRA case and will therefore not be treated explicitly again.

Among the most exciting schemes rank superstring induced scenarios (see e.g [21, 12, 13] and references therein). In this report a string effective field theory, based on orbifold compactification of the heterotic string, will be analyzed in which, though dominated by the vacuum expectation values of the dilaton field, supersymmetry breaking is also affected by the moduli fields. Such a mechanism gives rise to gaugino mass parameters with small but noticeable departure from universality, and non-universal sfermion mass parameters. From these mass parameters the fundamental parameters of the string effective field theory, such as the vacuum expectation values of the dilaton and the moduli fields, the moduli/dilaton mixing angle as well as the modular weights can be derived. In this way high-precision experiments can provide access to elements which are directly induced by superstrings [22].

Extrapolations over many orders of magnitude from the electroweak scale to scales near the Planck scale require high-precision measurements at the laboratory scale [23]. Such extrapolations can be performed in practice as demonstrated in the analysis of the electroweak and strong couplings at LEP and elsewhere [4. The unification of these couplings provides the most compelling argument, derived from experiment, in support of supersymmetry. An initial set of precision data on supersymmetric particles is expected from LHC experiments if favourable cascade decays can be exploited to measure mass differences very precisely [15]. A globally comprehensive high-precision analysis can only be performed at lepton colliders [24, 25, 16, 17, 26]. They are expected to be realized in a first phase up to an energy of about $1 \mathrm{TeV}$, and in a subsequent second phase up to about $5 \mathrm{TeV} \cdot e^{+} e^{-}$ linear collider designs for the first phase are being worked out for JLC, NLC and TESLA while the second phase may be realized in the CLIC technology. TESLA, in particular, can be operated at very high luminosity. A large number of threshold scans can therefore be performed which allows model independent high-precision measurements of the masses of supersymmetric particles. Chargino, neutralino and slepton masses are expected to be measured with accuracies at the per-mille level. Very heavy squarks and gluinos, on the other hand, may be analyzed in detail at CLIC after their discovery and first analysis at LHC. However, the accuracy is presumably reduced to the per-cent level as a consequence of the decreasing production cross sections, the non-zero widths of the heavy particles and the increasing energy smearing due to beam-strahlung.

Starting with observed numbers at the electroweak scale, the bottom-up approach exhausts all experimental information to the maximal extent possible in the empirical reconstruction of the underlying supersymmetric theory at the high scale. Finally, the parameters of the fundamental high-scale theory will become accessible in this way. This exploration of GUT and Planck scale physics by combining high precision with high energy in experiments at hadron and lepton colliders, is complemented by only a very small number of other methods, notabene proton decay, likely neutrino physics, textures of mass matrices, 
and cosmology. In all these individual approaches only scarce information on the underlying physical theoretical structures at the GUT/Planck scale can be extracted. Any of these methods should therefore be exploited in the maximal form in order to shed light on the boundaries of the physics area where gravity may affect properties and interactions of particles observed in the laboratory at the electroweak scale. In this way consequences of incorporating the fourth of the fundamental forces into the particle system could become accessible at laboratory experiments.

\section{Gravity Mediated SUSY Breaking}

\subsection{Minimal Supergravity - mSUGRA}

Supersymmetry cannot be broken spontaneously in our eigen-world without risking conflict with experimental results. The Ferrara-Girardello-Palumbo mass sum rule [27] requires supersymmetric particle masses below the corresponding Standard Model particle masses in this case - in obvious disagreement with observations. The elegant concept of spontaneous symmetry breaking, by non-perturbative gluino condensation for instance, can be realized, however, in a hidden sector which interacts with our eigen-world only by gravity. Gravitational interactions generate the soft supersymmetry breaking terms near the Grand Unification scale / Planck scale. Not compulsory but suggestive, the soft terms may be universal, i.e. the gaugino mass parameters and the scalar mass parameterst. Being flavour blind, the suppression of flavour-changing neutral processes can be realized in a natural way. Moreover, for a heavy top mass $m_{t} \simeq 174 \mathrm{GeV}$ the breaking of the electroweak symmetry $S U(2)_{L} \times U(1)_{Y} \rightarrow U(1)_{E M}$ can be generated radiatively. While at the universality scale all scalar masses squared are positive, the Higgs mass parameter $M_{H_{2}}^{2}$ turns negative at a scale of about $10^{4} \mathrm{TeV}$. This induces spontaneous electroweak symmetry breaking at the electroweak scale where the sum $M_{H_{2}}^{2}+|\mu|^{2}$ becomes negative, leaving however the strong and electromagnetic gauge symmetries $S U(3)_{C}$ and $U(1)_{E M}$ unbroken.

The minimal supergravity scenario mSUGRA is characterized by the universal parameters

$\begin{array}{llc}\text { gaugino mass parameter } & : & M_{1 / 2} \\ \text { scalar mass parameter } & : & M_{0} \\ \text { trilinear coupling } & : & A_{0}\end{array}$

complemented by the phase of $\mu$, the modulus $|\mu|$ determined by radiative symmetry breaking, and the mixing angle $\tan \beta$ in the Higgs sector. The mass parameters $M_{1 / 2}, M_{0}$ and the trilinear coupling $A_{0}$ are defined to be universal at the Grand Unification scale $M_{U}$. The unified gauge coupling is denoted by $\alpha_{U}$ at $M_{U}$, for the sake of simplicity taken real. These

\footnotetext{
${ }^{1}$ Universality may be broken GUT-scale threshold corrections, see e.g. Ref.[28]. The bottom-up approach should enable us to explore this domain in a systematic way to the maximum extent possible.
} 
parameters at the GUT scale are related to the low energy parameters by the supersymmetric renormalization group equations [29, 30] which to leading order generate the evolution for

$$
\begin{array}{lll}
\text { gauge couplings } & : & \alpha_{i}=Z_{i} \alpha_{U} \\
\text { gaugino mass parameters } & : & M_{i}=Z_{i} M_{1 / 2} \\
\text { scalar mass parameters } & : & M_{\tilde{j}}^{2}=M_{0}^{2}+c_{j} M_{1 / 2}^{2}+\sum_{\beta=1}^{2} c_{j \beta}^{\prime} \Delta M_{\beta}^{2} \\
\text { trilinear couplings } & : & A_{k}=d_{k} A_{0}+d_{k}^{\prime} M_{1 / 2}
\end{array}
$$

The index $i$ runs over the gauge groups $i=S U(3), S U(2), U(1)$. To leading order, the gauge couplings, and the gaugino and scalar mass parameters of soft-supersymmetry breaking depend on the $Z$ transporters

$$
Z_{i}=\left[1+b_{i} \frac{\alpha_{U}}{4 \pi} \log \left(\frac{M_{U}}{M_{Z}}\right)^{2}\right]^{-1}
$$

with $b\left[S U_{3}, S U_{2}, U_{1}\right]=-3,1,33 / 5$; the scalar mass parameters depend also on the Yukawa couplings $h_{t}, h_{b}, h_{\tau}$ of the top quark, bottom quark and $\tau$ lepton. The coefficients $c_{j}[j=$ $\left.L_{l}, E_{l}, Q_{l}, U_{l}, D_{l}, H_{1,2} ; l=1,2,3\right]$ for the slepton and squark doublets/singlets of generation $l$, and for the two Higgs doublets are linear combinations of the evolution coefficients $Z_{i}$; the coefficients $c_{j \beta}^{\prime}$ are of order unity. The shifts $\Delta M_{\beta}^{2}$ are nearly zero for the first two families of sfermions but they can be rather large for the third family and for the Higgs mass parameters, depending on the coefficients $Z_{i}$, the universal parameters $M_{0}^{2}, M_{1 / 2}$ and $A_{0}$, and on the Yukawa couplings $h_{t}, h_{b}, h_{\tau}$. The coefficients $d_{k}$ of the trilinear couplings $A_{k}[k=t, b, \tau]$ depend on the corresponding Yukawa couplings and they are approximately unity for the first two generations while being $\mathrm{O}\left(10^{-1}\right)$ and smaller if the Yukawa couplings are large; the coefficients $d_{k}^{\prime}$, depending on gauge and Yukawa couplings, are of order unity. Beyond the approximate solutions shown explicitly, the evolution equations have been solved numerically in the present analysis to two-loop order [30] and threshold effects have been incorporated at the low scale [31].

These parameters enter the mass-matrices for the various particles. In the case of charginos $\tilde{\chi}_{m}^{+}[m=1,2]$ the $2 \times 2$ mass matrix reads as

$$
M_{\tilde{\chi}^{+}}=\left(\begin{array}{cc}
M_{2} & \sqrt{2} m_{W} \cos \beta \\
\sqrt{2} m_{W} \sin \beta & \mu
\end{array}\right)
$$

while the mass matrix for neutralinos $\tilde{\chi}_{n}^{0}[n=1, . ., 4]$ is a $4 \times 4$ matrix,

$$
M_{\tilde{\chi}^{0}}=\left(\begin{array}{cccc}
M_{1} & 0 & -m_{Z} \cos \beta \sin \theta_{W} & m_{Z} \sin \beta \sin \theta_{W} \\
0 & M_{2} & m_{Z} \cos \beta \cos \theta_{W} & -m_{Z} \sin \beta \cos \theta_{W} \\
-m_{Z} \cos \beta \sin \theta_{W} & m_{Z} \cos \beta \cos \theta_{W} & 0 & -\mu \\
m_{Z} \sin \beta \sin \theta_{W} & -m_{Z} \sin \beta \cos \theta_{W} & -\mu & 0
\end{array}\right)
$$


Exploiting all the information available from a linear collider, both mass matrices can be reconstructed even the in case of complex parameters [32]. For large values, $\tan \beta$ needs supplementary analyses in the Higgs sector [33].

Assuming that the sfermion generations mix only weakly the mass matrices of the third generation sfermions can be written as:

$$
M_{\tilde{f}}^{2}=\left(\begin{array}{cc}
m_{\tilde{f}_{L}}^{2} & a_{f} m_{f} \\
a_{f} m_{f} & m_{\tilde{f}_{R}}^{2}
\end{array}\right)
$$

with

$$
\begin{aligned}
& m_{\tilde{f}_{L}}^{2}=M_{\tilde{F}_{L}}^{2}+\left(T_{f}^{3}-e_{f} \sin ^{2} \theta_{W}\right) \cos 2 \beta m_{Z}^{2}+m_{f}^{2}, \\
& m_{\tilde{f}_{R}}^{2}=M_{\tilde{F}_{R}}^{2}+e_{f} \sin ^{2} \theta_{W} \cos 2 \beta m_{Z}^{2}+m_{f}^{2}, \\
& a_{t} \equiv A_{t}-\mu \cot \beta, a_{b} \equiv A_{b}-\mu \tan \beta, a_{\tau} \equiv A_{\tau}-\mu \tan \beta
\end{aligned}
$$

where $e_{f}$ and $T_{f}^{3}$ are the electric charge and the third component of the weak isospin of the sfermion $\tilde{f} ; M_{\tilde{F}_{L}}=M_{\tilde{Q}}$ for $\tilde{f}_{L}=\tilde{t}_{L}, \tilde{b}_{L}, M_{\tilde{F}}=M_{\tilde{L}}$ for $\tilde{f}_{L}=\tilde{\tau}_{L}, \tilde{\nu}_{\tau} ; M_{\tilde{F}_{R}}=M_{\tilde{U}}, M_{\tilde{D}}, M_{\tilde{E}}$ for $\tilde{f}_{R}=\tilde{t}_{R}, \tilde{b}_{R}, \tilde{\tau}_{R}$, respectively; $m_{f}$ is the mass of the corresponding fermion. Also in this case it has been shown that the mass matrix can be reconstructed [34, 35]. The mass matrices for the first two generation sfermions have the same structure. However, due to the small fermion masses the mixing between the $L / R$ sfermions can be neglected in general. In the fits for the parameters we have used the complete one-loop mass matrices as given in [31. For the Higgs bosons also the two-loop contributions [36] are included.

The mSUGRA point we have analyzed in detail, was chosen close to the Snowmass Point SPS\#1 [18], except for the scalar mass parameter $M_{0}$ which was taken slightly larger for merely illustrative purpose: $M_{1 / 2}=250 \mathrm{GeV}, M_{0}=200 \mathrm{GeV}, A_{0}=-100 \mathrm{GeV}, \tan \beta=10$ and $\operatorname{sign}(\mu)=+$. The initial "experimental" values, have been generated by evolving the universal parameters down to the electroweak scale according to standard procedures [37, 31.

The parameters chosen are compatible with the present results of low-energy experiments which they affect by virtual contributions, and they are also compatible with dark-matter estimates [38]: $\operatorname{BR}(b \rightarrow s \gamma)=2.7 \cdot 10^{-4}, \Delta[g-2]_{\mu}=17 \cdot 10^{-10}, \Delta \rho=38 \cdot 10^{-5}$ and $\Omega h^{2}=0.4$. We have used the formulas given in [39] for the computation of $b \rightarrow s \gamma$, those given in 40] for $\Delta \rho$ and those given in [41] for $\Delta[g-2]_{\mu}$. $\Omega h^{2}$ has been calculated using the program of Ref. 42.

The five basic parameters define the experimental observables, including supersymmetric particle masses and production cross sections. They are endowed with errors as expected for threshold scans as well as measurements in the continuum at $e^{+} e^{-}$linear colliders (LC). Major parts of the LC analysis can be performed for energies below $1 \mathrm{TeV}$, some of the squarks require energies above $1 \mathrm{TeV}$. Estimates are based on integrated LC luminosities 
Table 1: Representative experimental mass errors used in the fits to the mass spectra; with the exception of the gluino mass, all the other parameters are based on LC measurements.

\begin{tabular}{c|cc||c|cc} 
Particle & $\mathrm{M}(\mathrm{GeV})$ & $\Delta \mathrm{M}(\mathrm{GeV})$ & Particle & $\mathrm{M}(\mathrm{GeV})$ & $\Delta \mathrm{M}(\mathrm{GeV})$ \\
\hline \hline$h^{0}$ & 113.33 & 0.05 & $\tilde{\nu}_{e L}$ & 256.79 & 0.11 \\
$H^{0}$ & 436.1 & 1.5 & $\tilde{e}_{L}$ & 269.1 & 0.3 \\
$A^{0}$ & 435.5 & 1.5 & $\tilde{e}_{R}$ & 224.82 & 0.15 \\
$H^{+}$ & 443.3 & 1.5 & $\tilde{\nu}_{\tau L}$ & 255.63 & 0.95 \\
$\tilde{\chi}_{1}^{+}$ & 183.05 & 0.15 & $\tilde{\tau}_{1}$ & 217.7 & 1.00 \\
$\tilde{\chi}_{2}^{+}$ & 383.3 & 0.3 & $\tilde{\tau}_{2}$ & 271.5 & 0.9 \\
$\tilde{\chi}_{1}^{0}$ & 97.86 & 0.2 & $\tilde{u}_{L}$ & 589 & 10 \\
$\tilde{\chi}_{2}^{0}$ & 184.6 & 0.3 & $\tilde{u}_{R}$ & 572 & 10 \\
$\tilde{\chi}_{3}^{0}$ & 365.5 & 0.3 & $\tilde{d}_{R}$ & 572 & 10 \\
$\tilde{\chi}_{4}^{0}$ & 383.0 & 0.7 & $\tilde{t}_{1}$ & 412 & 10 \\
$\tilde{g}$ & 598 & 10 & $\tilde{t}_{2}$ & 600 & 10 \\
\hline
\end{tabular}

of $1 \mathrm{ab}^{-1}$. The errors given in Ref. 25 are scaled in proportion to the masses of the spectrum. Typical examples are shown in Table 1. The LC errors on the squark masses, see e.g. Ref. 43], are set to an average value of $10 \mathrm{GeV}$ [similar errors may also be obtained if the precisely measured mass differences at the LHC are combined with high-precision measurements of the low-lying states at the LC]; varying this error within a factor two does not change the conclusions significantly since the measurement of the cross sections provides the maximal sensitivity in this sector. For the cross-sections we use purely statistical errors, while assuming a (conservative) reconstruction efficiency of $20 \%$. In addition the mass errors on the lightest gauginos were inflated with respect to earlier analyses to be conservative in advance of detailed experimental analyses of models with higher values of $\tan \beta$. [Parameter combinations from the fits to the spectrum and the cross sections which lead to charge and/or color breaking minima [44], are not accepted.] These observables are interpreted as the experimental input values for the evolution of the mass parameters in the bottom-up approach to the Grand Unification scale.

\subsubsection{Gauge Couplings}

The presumably strongest support, though indirect, for supersymmetry is related to the tremendous success of this theory in predicting the unification of the gauge couplings 4 . The precision, being at the per-cent level, is surprisingly high after extrapolations over fourteen orders of magnitude in the energy from the electroweak scale to the unification scale $M_{U}$. Conversely, the electroweak mixing angle has been predicted in this approach at the per-mille level. The evolution of the gauge couplings from low energy to the GUT scale 
$M_{U}$ is carried out in the two-loop accuracy. The gauge couplings $g_{1}, g_{2}, g_{3}$ and the Yukawa couplings are calculated in the $\overline{D R}$ scheme by adopting the shifts given in [31]. These parameters are evolved to $M_{U}$ using 2-loop RGEs [30]. At 2-loop order the gauge couplings do not meet exactly [45, 46], the differences owing to threshold effects at the unification scale $M_{U}$ which leave us with an ambiguity in the definition of $M_{U}$. In this report we define $M_{U}$ as the scale, ad libitum, where $g_{1}=g_{2}$ in the RGE evolution. The non-zero difference $g_{1}-g_{3}$ at this scale is then attributed to threshold effects of particles with masses of order $M_{U}$. The quantitative evolution implies important constraints on the particle content at $M_{U}$ [47-51].

Based on the set of low-energy gauge and Yukawa parameters $\left\{\alpha\left(m_{Z}\right), \sin ^{2} \theta_{W}, \alpha_{s}\left(m_{Z}\right)\right.$, $\left.Y_{t}\left(m_{Z}\right), Y_{b}\left(m_{Z}\right), Y_{\tau}\left(m_{Z}\right)\right\}$ the evolution of the inverse couplings $\alpha_{i}^{-1}[i=U(1), S U(2)$, $S U(3)$ is depicted in Fig. 17a. The evolution is performed for the mSUGRA reference point defined above. Unlike earlier analyses, the low-energy thresholds of supersymmetric particles can be calculated in this framework exactly without reference to effective SUSY scales. The broken error ellipse in Fig. 1 $1 \mathrm{~b}$, derived for $\left[M_{U}, \alpha_{U}\right]$ by requiring $g_{1}=g_{2}$, corresponds to the present experimental accuracy of the gauge couplings [52]: $\Delta\left\{\alpha^{-1}\left(m_{Z}\right), \sin ^{2} \theta_{W}\right.$, $\left.\alpha_{s}\left(m_{Z}\right)\right\}=\left\{0.03,1.7 \cdot 10^{-4}, 3 \cdot 10^{-3}\right\}$. The full ellipse demonstrates the improvement for the absolute errors $\left\{10^{-3}, 10^{-5}, 10^{-3}\right\}$ after operating GigaZ [53, 54]. The expected accuracies in $M_{U}$ and $\alpha_{U}$ are summarized in the values given in Table 2. The difference between the unification point in the ellipse and the value of $g_{3}$ is accounted for by contributions from high scale physics, colour-triplet Higgs fields, for example. Thus, for a typical set of SUSY parameters, the evolution of the gauge couplings from low to high scales leads to a precision of 1.5 per-cent of the Grand Unification picture.

Table 2: Expected errors on $M_{U}$ and $\alpha_{U}$ for the $m S U G R A$ reference point, derived for the present level of accuracy and compared with GigaZ.

\begin{tabular}{c||c|c} 
& Now & GigaZ \\
\hline \hline$M_{U}$ & $(2.00 \pm 0.06) \cdot 10^{16} \mathrm{GeV}$ & $(2.000 \pm 0.016) \cdot 10^{16} \mathrm{GeV}$ \\
$\alpha_{U}^{-1}$ & $24.364 \pm 0.015$ & $24.361 \pm 0.007$ \\
\hline
\end{tabular}

\subsubsection{Gaugino and Scalar Mass Parameters}

The results for the evolution of the mass parameters to the GUT scale $M_{U}$ are shown in Fig. 2. Fig. 2(a) presents the evolution of the gaugino parameters $M_{i}^{-1}$ which clearly is under excellent control, as is the extrapolation of the slepton mass parameter squared of the first (and second) and the third generation in Fig. 2(c) and (d), respectively. The accuracy deteriorates for the squark mass parameters and for the Higgs mass parameter $M_{H_{2}}^{2}$. The 
a)

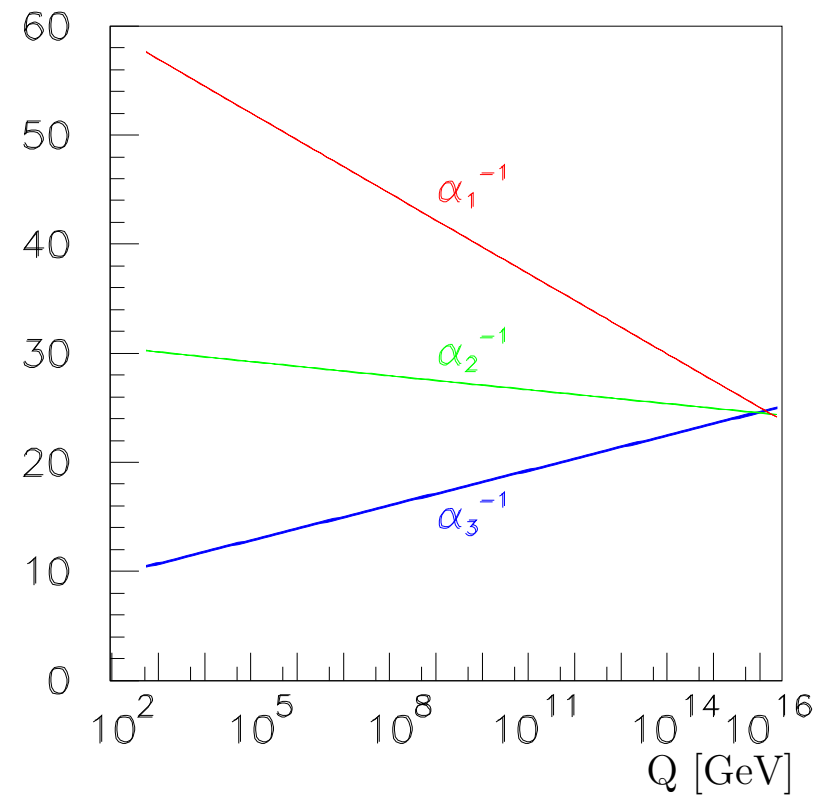

b)

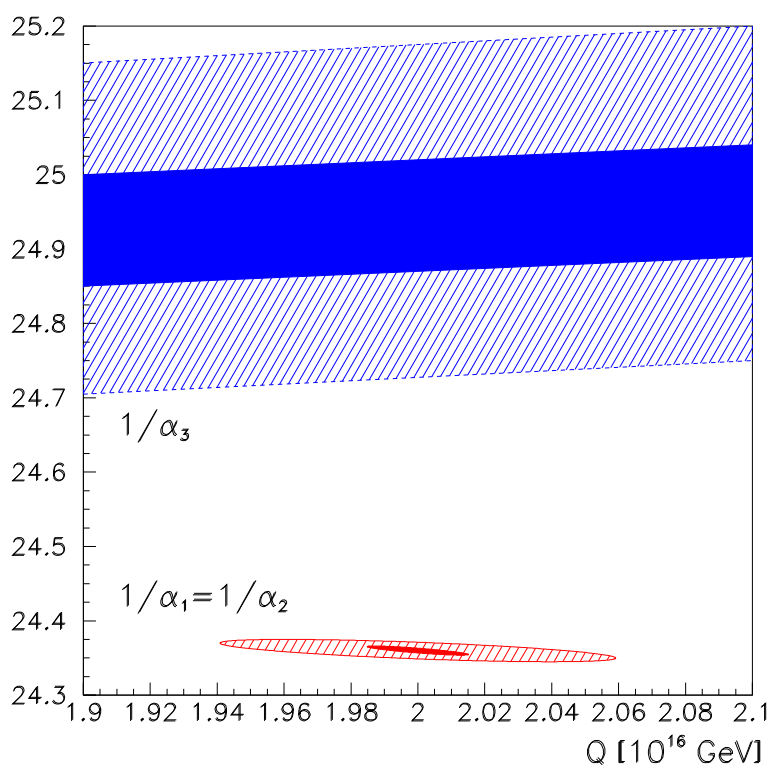

Figure 1: a) Running of the inverse gauge couplings. b) Determination of $M_{U}, \alpha_{U}^{-1}$; the unification point $U$ is defined by the meeting point of $\alpha_{1}$ with $\alpha_{2}$. The wide error bands are based on present data, the narrow bands demonstrate the improvement expected by future GigaZ analyses.

origin of the differences between the errors for slepton, squark and Higgs mass parameters can be traced back to the numerical size of the coefficients in Eqs. (3). Typical examples using the formulas presented in Appendix B evaluated at $Q=500 \mathrm{GeV}$ read as follows:

$$
\begin{aligned}
M_{\tilde{L}_{1}}^{2} & \simeq M_{0}^{2}+0.47 M_{1 / 2}^{2} \\
M_{\tilde{Q}_{1}}^{2} & \simeq M_{0}^{2}+5.0 M_{1 / 2}^{2} \\
M_{\tilde{H}_{2}}^{2} & \simeq-0.03 M_{0}^{2}-1.34 M_{1 / 2}^{2}+1.5 A_{0} M_{1 / 2}+0.6 A_{0}^{2} \\
|\mu|^{2} & \simeq 0.03 M_{0}^{2}+1.17 M_{1 / 2}^{2}-2.0 A_{0} M_{1 / 2}-0.9 A_{0}^{2}
\end{aligned}
$$

While the coefficients for sleptons are of order unity, the coefficient $c_{j}$ for squarks grows very large, $c_{j} \simeq 5$.0, so that small errors in $M_{1 / 2}^{2}$ are magnified by nearly an order of magnitude in the solution for $M_{0}$. By close inspection of Eq.(3) for the Higgs mass parameter it turns out that the formally leading $M_{0}^{2}$ part is nearly cancelled by the $M_{0}^{2}$ part of $c_{j, \beta}^{\prime} \Delta M_{\beta}^{2}$. Inverting Eq.(3) for $M_{0}^{2}$ therefore gives rise to large errors in the Higgs case. A representative set of mass values and the associated errors, after evolution from the electroweak scale to $M_{U}$, is presented in Table 3. The corresponding error ellipses for the unification of the gaugino masses are shown in Fig. 2(b).

Extracting the trilinear parameters $A_{k}$ is difficult and more refined analyses based on sfermion cross sections and Higgs and/or sfermion decays are necessary to determine these 
Table 3: Representative gaugino/scalar mass parameters and couplings as determined at the electroweak scale and evolved to the GUT scale in the mSUGRA scenario; based on LHC and LC simulations. $M_{L_{1,3}}^{2}, M_{Q_{1,3}}^{2}$ are the slepton and squark isodoublet parameters of the first and third family whereas $M_{E_{1,3}}^{2}, M_{U_{1,3}}^{2}$ and $M_{D_{1,3}}^{2}$ are the the slepton and squark isosinglet parameters of the first and third family. [The errors quoted correspond to $1 \sigma$.

\begin{tabular}{|c|c|c|}
\hline & Exp. Input & GUT Value \\
\hline$M_{1}[\mathrm{GeV}]$ & $102.31 \pm 0.25$ & $250.00 \pm 0.33$ \\
\hline$M_{2}[\mathrm{GeV}]$ & $192.24 \pm 0.48$ & $250.00 \pm 0.52$ \\
\hline$M_{3}[\mathrm{GeV}]$ & $586 \pm 12$ & $250.0 \pm 5.3$ \\
\hline$\mu$ & $358.23 \pm 0.28$ & $355.6 \pm 1.2$ \\
\hline$M_{L_{1}}^{2}\left[\mathrm{GeV}^{2}\right]$ & $(6.768 \pm 0.005) \cdot 10^{4}$ & $(3.99 \pm 0.41) \cdot 10^{4}$ \\
\hline$M_{E_{1}}^{2}\left[\mathrm{GeV}^{2}\right]$ & $(4.835 \pm 0.007) \cdot 10^{4}$ & $(4.02 \pm 0.82) \cdot 10^{4}$ \\
\hline$M_{Q_{1}}^{2}\left[\mathrm{GeV}^{2}\right]$ & $(3.27 \pm 0.08) \cdot 10^{5}$ & $(3.9 \pm 1.5) \cdot 10^{4}$ \\
\hline$M_{U_{1}}^{2}\left[\mathrm{GeV}^{2}\right]$ & $(3.05 \pm 0.11) \cdot 10^{5}$ & $(3.9 \pm 1.9) \cdot 10^{4}$ \\
\hline$M_{D_{1}}^{2}\left[\mathrm{GeV}^{2}\right]$ & $(3.05 \pm 0.11) \cdot 10^{5}$ & $(4.0 \pm 1.9) \cdot 10^{4}$ \\
\hline$M_{L_{3}}^{2}\left[\mathrm{GeV}^{2}\right]$ & $(6.711 \pm 0.050) \cdot 10^{4}$ & $(4.00 \pm 0.41) \cdot 10^{4}$ \\
\hline$M_{E_{3}}^{2}\left[\mathrm{GeV}^{2}\right]$ & $(4.700 \pm 0.087) \cdot 10^{4}$ & $(4.03 \pm 0.83) \cdot 10^{4}$ \\
\hline$M_{Q_{3}}^{2}\left[\mathrm{GeV}^{2}\right]$ & $(2.65 \pm 0.10) \cdot 10^{5}$ & $(4.1 \pm 3.0) \cdot 10^{4}$ \\
\hline$M_{U_{3}}^{2}\left[\mathrm{GeV}^{2}\right]$ & $(1.86 \pm 0.12) \cdot 10^{5}$ & $(4.0 \pm 3.6) \cdot 10^{4}$ \\
\hline$M_{D_{3}}^{2}\left[\mathrm{GeV}^{2}\right]$ & $(3.03 \pm 0.12) \cdot 10^{5}$ & $(4.0 \pm 2.6) \cdot 10^{4}$ \\
\hline$M_{H_{1}}^{2}\left[\mathrm{GeV}^{2}\right]$ & $(6.21 \pm 0.08) \cdot 10^{4}$ & $(4.01 \pm 0.54) \cdot 10^{4}$ \\
\hline$M_{H_{2}}^{2}\left[\mathrm{GeV}^{2}\right]$ & $(-1.298 \pm 0.004) \cdot 10^{5}$ & $(4.1 \pm 3.2) \cdot 10^{4}$ \\
\hline$A_{t}[\mathrm{GeV}]$ & $-446 \pm 14$ & $-100 \pm 54$ \\
\hline $\tan \beta$ & $9.9 \pm 0.9$ & - \\
\hline
\end{tabular}

parameters accurately. $A_{t}$ can be obtained from the mixing angle of the stop sector by measuring the stop production cross section in $e^{+} e^{-}$annihilation with different electron and/or positron polarizations [35]. In the cases $A_{b}$ and $A_{\tau}$ the situation is more difficult, because these parameters influence the mixing angle in the sbottom and stau sector only weakly as evident from Eq.(11). In these cases the $\tilde{b}$ and $\tilde{\tau}$ couplings to the Higgs bosons must be measured, because these couplings include terms directly proportional to $A_{k} \tan \beta$. For instance, by analysing the decays $\tilde{\tau}_{2} \rightarrow A^{0} \tilde{\tau}_{1}, h^{0} \tilde{\tau}_{1}$ and $H^{0} \tilde{\tau}_{1}, A_{\tau}$ can be extracted within $10 \%$ [34]. If these modes are kinematically forbidden, the couplings can either be measured in the decays of the heavier Higgs bosons, as $H^{0}, A^{0} \rightarrow \tilde{\tau}_{1} \tilde{\tau}_{1}$, or by means of the cross sections for processes such as $e^{+} e^{-} \rightarrow \tilde{\tau}_{1} \tilde{\tau}_{1} h^{0}$. Similar procedures are expected to apply for $A_{b}$. In certain areas of SUSY parameter space, the trilinear couplings can also be extracted from measurements of the degree of fermion polarization [55] in sfermion decays $\tilde{t}, \tilde{b}$ and $\tilde{\tau}$. 
(a)

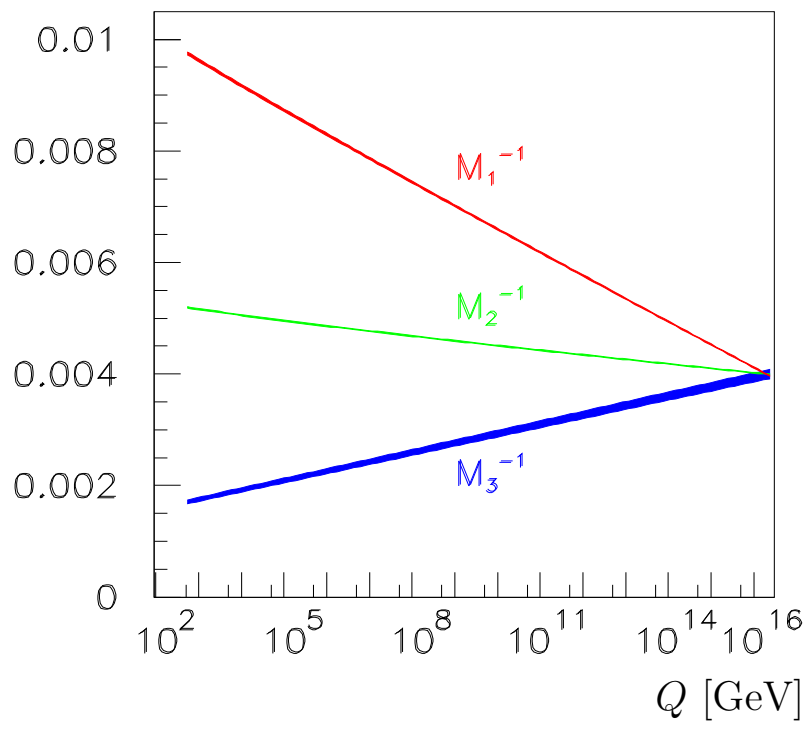

(b)

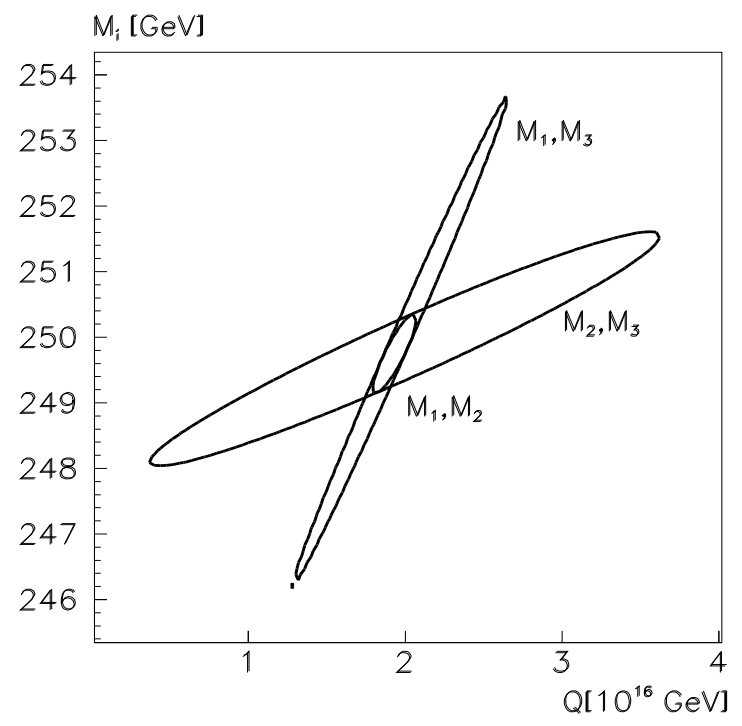

(c)

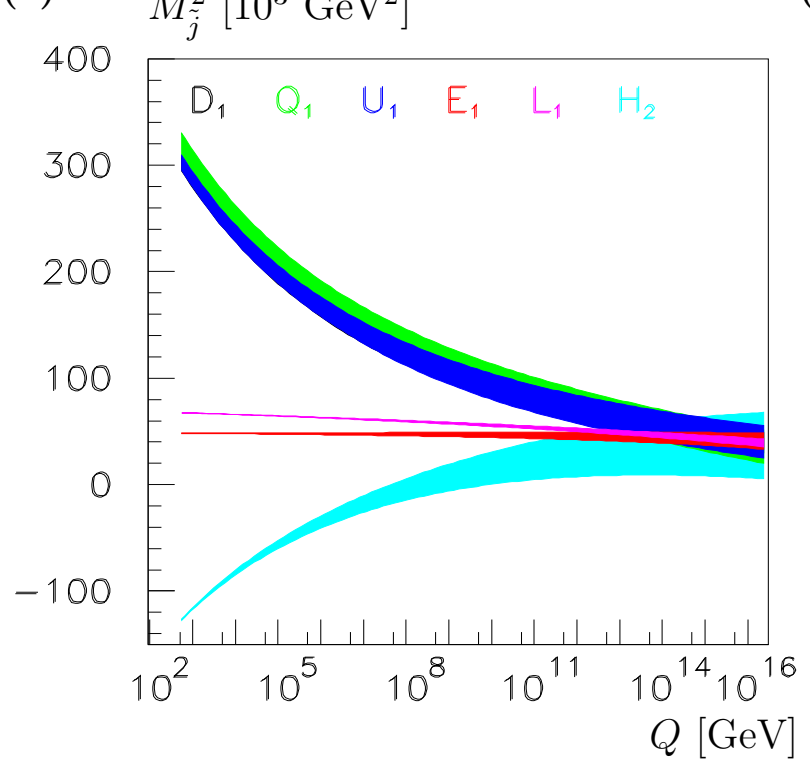

(d)

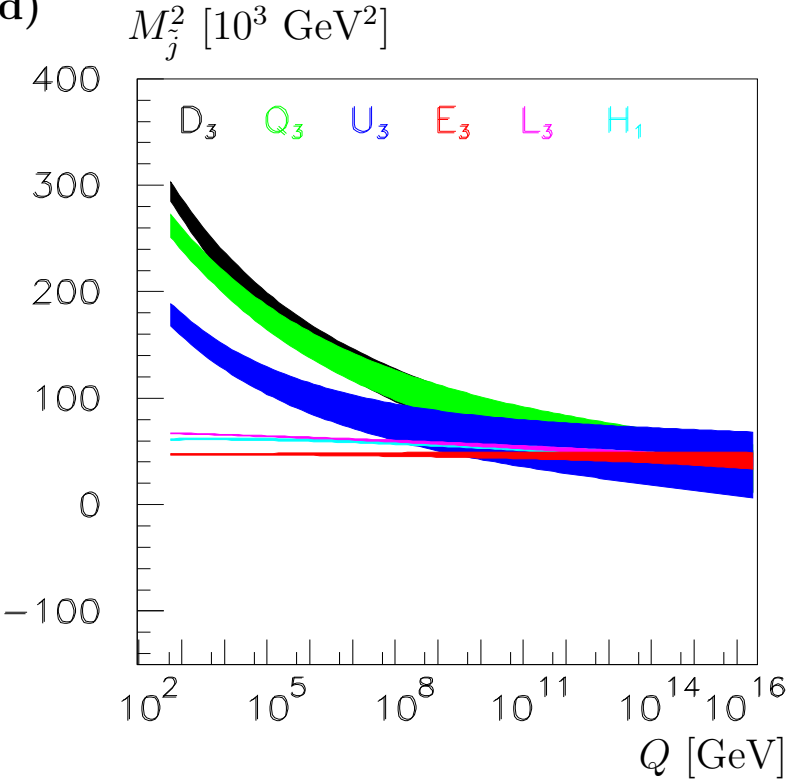

Figure 2: mSUGRA: Evolution, from low to high scales, of (a) gaugino mass parameters, and (b) unification of gaugino mass parameter pairs; (c) evolution of first-generation sfermion mass parameters and the Higgs mass parameter $M_{H_{2}}^{2}$; (d) evolution of thirdgeneration sfermion mass parameters and the Higgs mass parameter $M_{H_{1}}^{2}$. The mSUGRA point probed is defined by the parameters $M_{0}=200 \mathrm{GeV}, M_{1 / 2}=250 \mathrm{GeV}, A_{0}=-100 \mathrm{GeV}$, $\tan \beta=10$, and $\operatorname{sign}(\mu)=(+)$. [The widths of the bands indicate the $1 \sigma C L$.] 
Table 4: Comparison of the ideal parameters with the experimental expectations for the particular mSUGRA reference point analyzed in this report. [All mass parameters are given in units of $\mathrm{GeV}$.]

\begin{tabular}{c||c|c} 
& Ideal & Experimental Error \\
\hline \hline$M_{U}$ & $2 \cdot 10^{16}$ & $1.6 \cdot 10^{14}$ \\
$\alpha_{U}^{-1}$ & 24.361 & 0.007 \\
\hline$M_{\frac{1}{2}}$ & 250 & 0.08 \\
$M_{0}$ & 200 & 0.09 \\
$A_{0}$ & -100 & 1.8 \\
\hline$\mu$ & 358.23 & 0.21 \\
$\tan \beta$ & 10 & 0.1 \\
\hline
\end{tabular}

The unified value $A_{0}$ of the $A_{t}$ coupling, the best measured coupling among the $A_{k}$ parameters, is shielded by the pseudo-fixed point behaviour of $A_{t}$ [56] since $d_{t} \simeq 0.2$ is small compared to $d_{t}^{\prime} \simeq 2$. The impact of the other trilinear couplings on physical observables is weak so that large experimental errors are expected. As a result, the universal character of the fundamental parameter $A_{0}$ cannot be determined as precisely as the other parameters at the GUT scale. Although the trilinear couplings $A_{b}$ and $A_{\tau}$ have only little impact on physical observables, they do strongly influence the running of the third generation sfermion mass parameters as well as the Higgs mass parameters. The error propagation is stabilized if $A_{\tau}$ and $A_{b}$ can be measured in the way outlined above. [Otherwise the errors would increase by an order of magnitude.] The detailed analysis in this report has been based on the auxiliary assumption that $A_{b}$ and $A_{\tau}$ are within $1 \sigma$ of $A_{t}=A_{0}$ at $M_{U}$; this assumption is conservative if the envisaged experimental analyses of $A_{\tau}$ and $A_{b}$ can be performed at the electroweak scale in practice.

Even though the auxiliary assumption seems conservative, given the size of the error on $A_{0}$ determined from $A_{t}$, dedicated phenomenological and experimental analyses of the third family must be developed, as indicated above, to improve the measurement of the associated parameters, in particular in view of the evolution of the Higgs mass parameter which induces electroweak symmetry breaking.

Inspecting Figs. 2(c) and (d) leads to the conclusion that a blind top-down approach eventually may generate an incomplete picture. Global fits based on mSUGRA without allowing for deviations from universality, are dominated by $M_{1,2}$ and the slepton mass parameters due to the pseudo-fixed point behaviour of the squark mass parameters. Therefore, the structure of the theory in the squark sector is not scrutinized stringently at the unification scale in the top-down approach let alone the Higgs sector. By contrast, the bottom-up approach demonstrates very clearly the extent to which the theory can be tested at the high 
scale quantitatively. The quality of the test is apparent from Table 3, in which the evolved gaugino values should reproduce the universal mass $M_{1 / 2}=250 \mathrm{GeV}$ and all the scalars the mass $M_{0}=200 \mathrm{GeV}$. They are compared with the global mSUGRA fit of the universal parameters in Table 4 .

\section{$2.2 \quad$ Left-Right Supergravity}

It is generally accepted that neutrinos are massive particles, though at a very low scale. Supersymmetric scenarios like MSSM and mSUGRA must therefore be extended to incorporate the right-handed neutrino degrees of freedom. Since the complexity grows strongly with the rising number of parameters, it is useful, in a first attempt, to analyze the system in characteristic scenarios based on compelling physical assumptions. In particular, we will assume that the small neutrino masses are generated by the seesaw mechanism 20]. Moreover, we will assume hierarchies for the heavy neutrino masses as well as the neutrino Yukawa couplings similar to the up-type particles in the quark sector; such a scheme, suggested by $S O(10) \mathrm{GUT}$, is compatible with the data collected in low-energy neutrino experiments [57].

This scenario can be embedded in a Grand Unified $S O(10)$ theory with the following breaking pattern of the symmetries. The $S O(10)$ symmetry is realized between the Planck scale $M_{P}$ and a scale $M_{S O(10)}$ at which the symmetry breaks to $S U(5)$. The scale $M_{S O(10)}$ is assumed above the scale $M_{U}$ where $S U(5)$ breaks to the symmetry group $S U(3)_{C} \times$ $S U(2)_{L} \times U(1)_{Y}$ of the Standard Model. At the scale $M_{U}$ the gauge couplings split and the effective theory is the MSSM plus right-handed neutrinos with masses of order $10^{9}$ to $10^{15} \mathrm{GeV}$. Below this mass scale the right-handed neutrinos freeze out and the MSSM is effectively realized in its standard form. The relevant SUSY parameters are summarized in Table 5. It is less obvious that $M_{U}$ associated with the $S U(5)$ symmetry is the scale where the gaugino and scalar mass parameters are universal. The supporting argument for this point is derived empirically from the unification of the gauge couplings. Nevertheless, the subsequent analysis will be based on this hypothesis which, of course, is a clear target for confirmation or rejection in the bottom-up approach we investigate?.

In this left-right supergravity point, called LR-SUGRA for short's, we have probed the same SUSY parameters as above, complemented by the same universal parameters in the right-handed sneutrino sector. The sneutrinos $\tilde{\nu}_{L}$ and $\tilde{\nu}_{R}$ mix by the (large) Yukawa interactions in the $\hat{\nu}_{R}$ sector of the superpotential to form the mass eigenstates $\tilde{\nu}_{1}$ and $\tilde{\nu}_{2}$. Also in this sector an effective seesaw mechanism is induced by the large $\nu_{R}$ mass, as can

\footnotetext{
${ }^{2}$ Potential sources of deviations from this picture can easily be illustrated by assuming $M_{S O(10)}$ as the scale proper of universality: The Yukawa interactions contribute differently to the running of the $M_{\widetilde{10}}^{2}, M_{\tilde{5}}^{2}$, $M_{\tilde{\nu}_{R}}^{2}$; the same holds true for the $A$ parameters [58]. Moreover, different $D$-term contributions to the scalar masses are in general generated by the breaking mechanism from $S O(10)$ to $S U(5)$ [59].

${ }^{3}$ Other left-right scenarios will be presented in a forthcoming publication.
} 


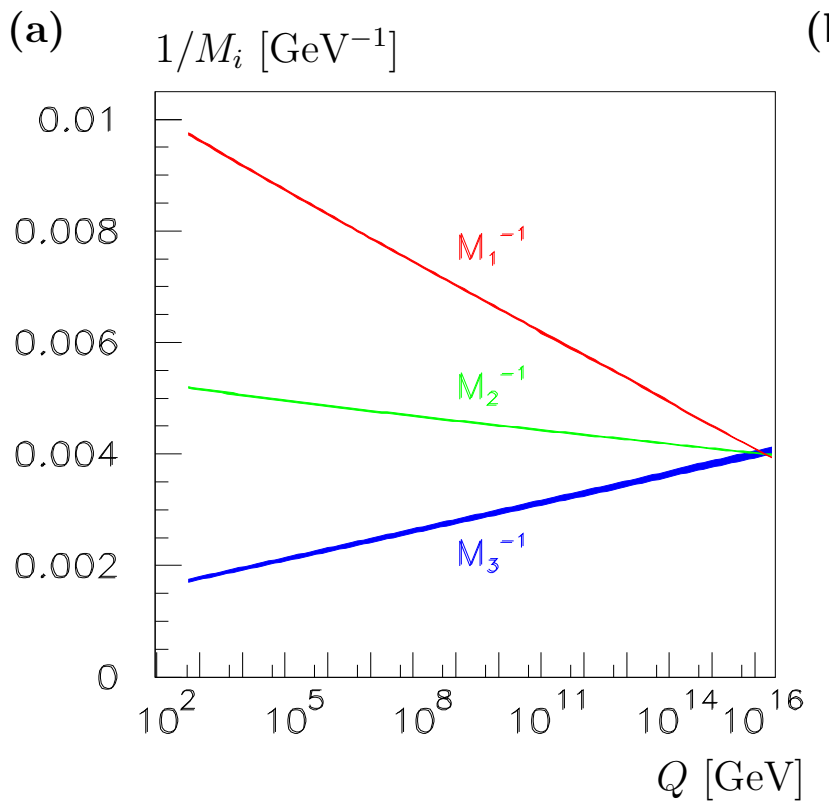

(b)
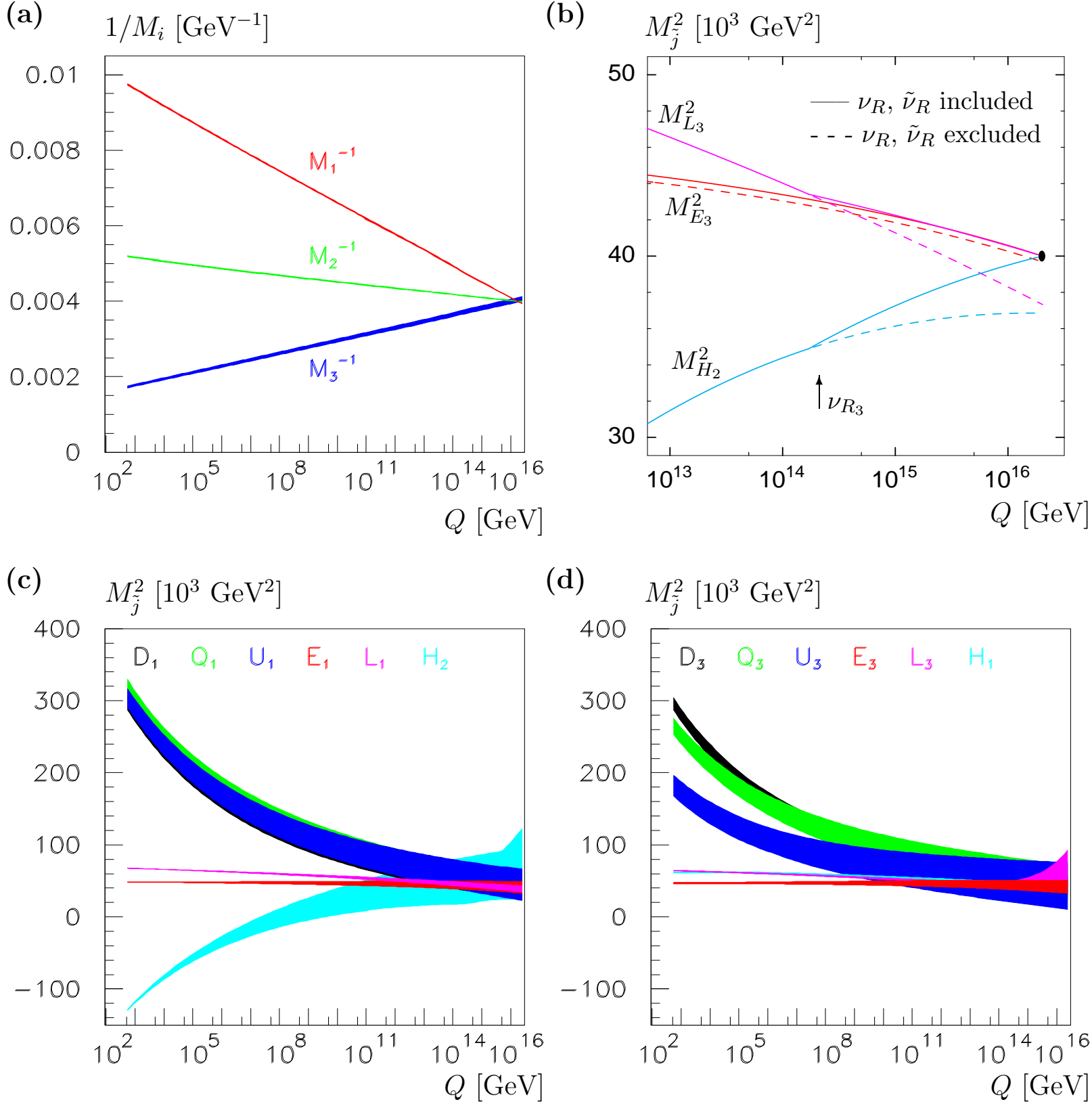

(d)

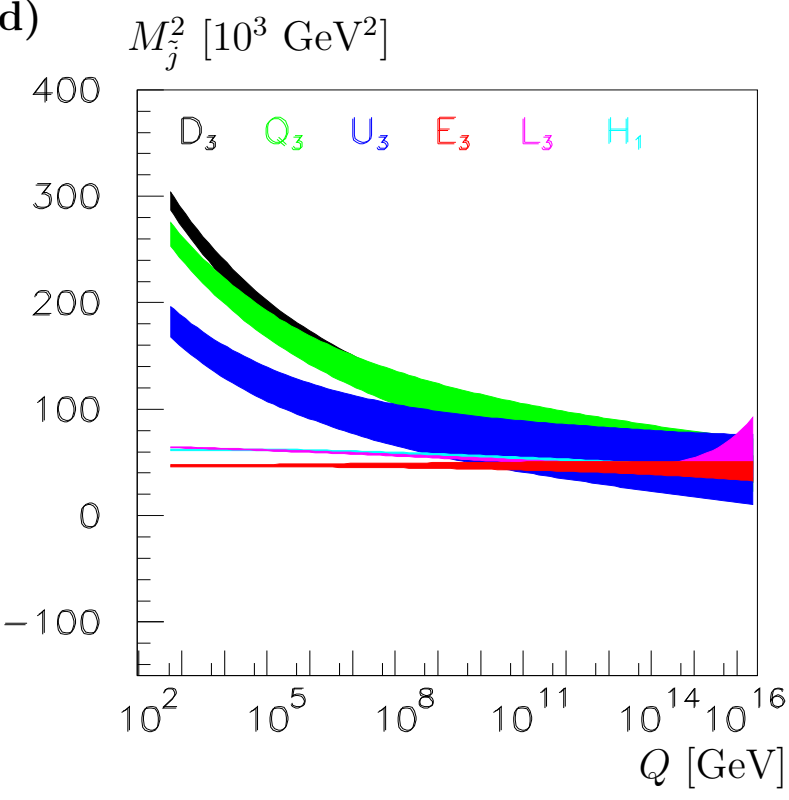

Figure 3: LR-SUGRA with $\nu_{R}$ : Evolution of (a) gaugino mass parameters, (b) evolution of third generation slepton mass parameters and Higgs mass parameters $M_{\mathrm{H}_{2}}^{2}$; (c) evolution of first-generation sfermion mass parameters and Higgs mass parameters $M_{\mathrm{H}_{2}}^{2}$; (d) evolution of third generation sfermion mass parameters and Higgs mass parameters $M_{H_{1}}^{2}$. The $m S U G R A$ point probed is characterized by the parameters $M_{0}=200 \mathrm{GeV}, M_{1 / 2}=250 \mathrm{GeV}$, $A_{0}=-100 \mathrm{GeV}, \tan \beta=10$, and $\operatorname{sign}(\mu)=(+)$. [The widths of the bands indicate the $1 \sigma$ $C L$.] 
Table 5: Scales and soft SUSY breaking parameters of the effective left-right supergravity theory analyzed in this report.

\begin{tabular}{l||l|l} 
Scale & Gauge Group & Parameters \\
\hline \hline$M_{P}-M_{S O(10)}$ & $S O(10)$ & $M_{1 / 2}, M_{\tilde{16}}^{2}, A_{0}$ \\
$M_{S O(10)}-M_{U}$ & $S U(5)$ & $M_{1 / 2}, M_{\widetilde{10}}^{2}, M_{\tilde{5}}^{2}$ \\
& & $M_{\tilde{\nu}}^{2}, A_{10}, A_{5}, A_{\nu}$ \\
$M_{U}-M_{\nu_{R}}$ & $S U(3)_{C} \otimes S U(2)_{L} \otimes U(1)_{Y}$ & $M_{1}, M_{2}, M_{3}$ \\
& & $M_{\tilde{Q}}^{2}, M_{\tilde{U}}^{2}, M_{\tilde{D}}^{2}$ \\
& & $M_{\tilde{L}}^{2}, M_{\tilde{\nu} R}^{2}, M_{\tilde{E}}^{2}$ \\
$M_{\nu_{R}}-M_{E W}$ & $S U(3)_{C} \otimes S U(2)_{L} \otimes U(1)_{Y}$ & $A_{u}, A_{d}, A_{\tau}, A_{\nu}$ \\
& & $M_{1}, M_{2}, M_{3}$ \\
& & $M_{\tilde{Q}}^{2}, M_{\tilde{U}}^{2}, M_{\tilde{D}}^{2}$ \\
& & $M_{\tilde{L}}^{2}, M_{\tilde{E}}^{2}$ \\
& & $A_{u}, A_{d}, A_{\tau}$ \\
\hline
\end{tabular}

be most easily seen by considering the one generation case:

$$
m^{2}=\left(\begin{array}{cc}
M_{\tilde{L}}^{2}+\frac{1}{2} m_{Z}^{2} \cos 2 \beta & \frac{1}{\sqrt{2}} Y_{\nu}\left(A_{\nu} v_{2}-\mu v_{1}\right) \\
\frac{1}{\sqrt{2}} Y_{\nu}\left(A_{\nu} v_{2}-\mu v_{1}\right) & M_{\tilde{\nu}_{R}}^{2}+M_{\nu_{R}}^{2}
\end{array}\right)
$$

In this mass matrix $M_{\nu_{R}}$ is the [GUT-scale] mass of the right-handed neutrino, $M_{\tilde{\nu}_{R}}$ the scalar [TeV-scale] mass parameter of the right sneutrino, $Y_{\nu}$ and $A_{\nu}$ the neutrino Yukawa coupling and the neutrino trilinear coupling, respectively. $v_{1}$ and $v_{2}$ are the vacuum expectation values of the Higgs field with isospin $-1 / 2$ and isospin $1 / 2$, respectively. The approximate eigenvalues of the sneutrino mass matrix read

$$
\begin{aligned}
& m_{\tilde{\nu}_{1}}^{2} \simeq M_{\tilde{L}}^{2}+\frac{1}{2} m_{Z}^{2} \cos 2 \beta-Y_{\nu}^{2} \frac{\left(A_{\nu} v_{2}-\mu v_{1}\right)^{2}}{2 M_{\nu_{R}}^{2}} \\
& m_{\tilde{\nu}_{2}}^{2} \simeq M_{\nu_{R}}^{2}+M_{\tilde{\nu}_{R}}^{2}+Y_{\nu}^{2} \frac{\left(A_{\nu} v_{2}-\mu v_{1}\right)^{2}}{2 M_{\nu_{R}}^{2}}
\end{aligned}
$$

Therefore, to a very good approximation, $\tilde{\nu}_{1}$ coincides with $\tilde{\nu}_{L}$ and $\tilde{\nu}_{2}$ with $\tilde{\nu}_{R}$. The heavy right-handed neutrino masses are calculated by identifying the Yukawa couplings with the up-type quark couplings in the quark sector at the GUT scale (largely equivalent to the $S O(10)$ scale in this regard) and by identifying the light neutrino masses with the neutrino mass differences in the large mixing angle for the solar neutrino problem solution: $m_{\nu_{L 1}}=$ $10^{-5} \mathrm{eV}, m_{\nu_{L 2}}=3 \cdot 10^{-3} \mathrm{eV}, m_{\nu_{L 3}}=6 \cdot 10^{-2} \mathrm{eV} ; M_{\nu_{R_{1}}}=3 \cdot 10^{9} \mathrm{GeV}, M_{\nu_{R_{2}}}=1.4 \cdot 10^{11} \mathrm{GeV}$, $M_{\nu_{R_{3}}}=1.7 \cdot 10^{14} \mathrm{GeV}$.

The impact on the evolution of the mass parameters is rather simple. In the analysis of the first two generations the Yukawa interactions involving the heavy neutrinos and the 
R-sneutrinos are so small that their effect is not noticeable in practice. The evolution of the gaugino and scalar mass parameters is not affected by the left-right extension of the system in the present form as is evident from Figs. 3(a) and (c). This is only different for the third generation and for $M_{H_{2}}^{2}$ owing to the enhanced Yukawa coupling in this case as shown in Figs. 3(b) and (d). The sensitivity to the intermediate $\nu_{R}$ scales is rather weak because neutrino Yukawa couplings affect the evolution of the sfermion mass parameters only mildly. Since the $\nu_{R}$ of the third generation is unfrozen only beyond $Q=M_{\nu_{R}}$ the impact of the LR extension becomes visible in the evolution only at very high scales. In Fig. 3(b) we display the evolution of $M_{\tilde{E}_{3}}^{2}, M_{\tilde{L}_{3}}^{2}$ and $M_{H_{2}}^{2}$ for illustrative purposes. The full lines include the effects of the right-handed neutrino, which are to be compared with the dashed lines where the $\nu_{R}$ effects are removed. The scalar mass parameter $M_{\tilde{E}_{3}}^{2}$ appears unaffected by the right-handed sector, while $M_{\tilde{L}_{3}}^{2}$ and $M_{H_{2}}^{2}$ clearly are. Only the picture including $\nu_{R}, \tilde{\nu_{R}}$ is compatible with the unification assumption. The kinks in the evolution of $M_{\tilde{L}_{3}}^{2}$ and $M_{\tilde{H}_{2}}^{2}$ can be traced back to the fact that around $10^{14} \mathrm{GeV}$ the third generation (s)neutrinos become quantum mechanically effective, given a large enough neutrino Yukawa coupling to influence the evolution of these mass parameters. A much better understanding of the third generation family must be achieved to draw quantitative conclusions beyond the rough estimates of the $\nu_{R}$ scales sketched in the present analysis.

\section{Gauge Mediated Supersymmetry Breaking}

Motivated by the observed suppression of flavour changing neutral transitions, supersymmetry breaking mediated by gauge interactions from a secluded sector to the visible eigenworld, offers an automatic solution to this problem [9, 60]. The scalar and the F components of a Standard-Model singlet superfield $S$ acquire vacuum expectation values $\langle S\rangle$ and $\left\langle F_{S}\right\rangle$ through interactions with fields in the secluded sector, thus breaking supersymmetry.f Vector-like messenger fields $M$, carrying non-zero $S U(3) \times S U(2) \times U(1)$ charges and coupling to $S$, transport the supersymmetry breaking to the eigen-world. The system is characterized by the mass $M_{M} \sim\langle S\rangle$ of the messenger fields and the mass scale $\Lambda=\left\langle F_{S}\right\rangle /\langle S\rangle$ setting the seize of the gaugino and scalar masses. $M_{M}$ is expected to be in the range of 10 to $10^{6} \mathrm{TeV}$ and $\Lambda$ has to be smaller than $M_{M}$. The gaugino masses

$$
M_{i}\left(M_{M}\right)=\left(N_{5}+3 N_{10}\right) g\left(\Lambda / M_{M}\right) \alpha_{i}\left(M_{M}\right) \Lambda
$$

\footnotetext{
${ }^{4}$ A solution of the doublet-triplet splitting problem can be found in GMSB by introducing two different $S$ fields. The masses of supersymmetric particles are less constrained in this approach than in the one-scale model, and they depend on the values of the two $\Lambda_{i}$ parameters. In particular, the approximate equality of the gaugino masses at the GUT scale is lifted, see 61 for details.
} 
(a)

$$
1 / M_{i}\left[\mathrm{GeV}^{-1}\right]
$$

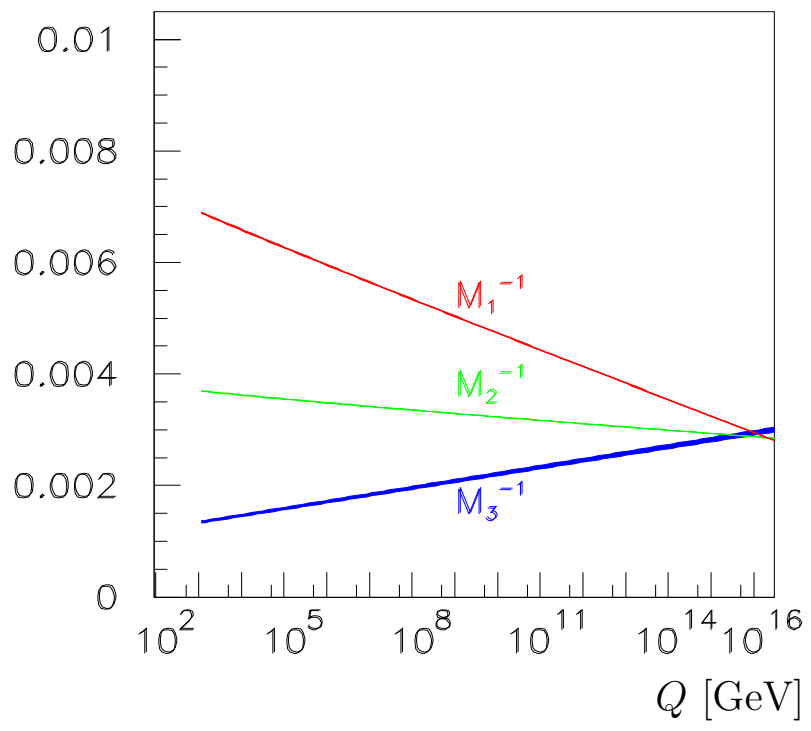

(c)

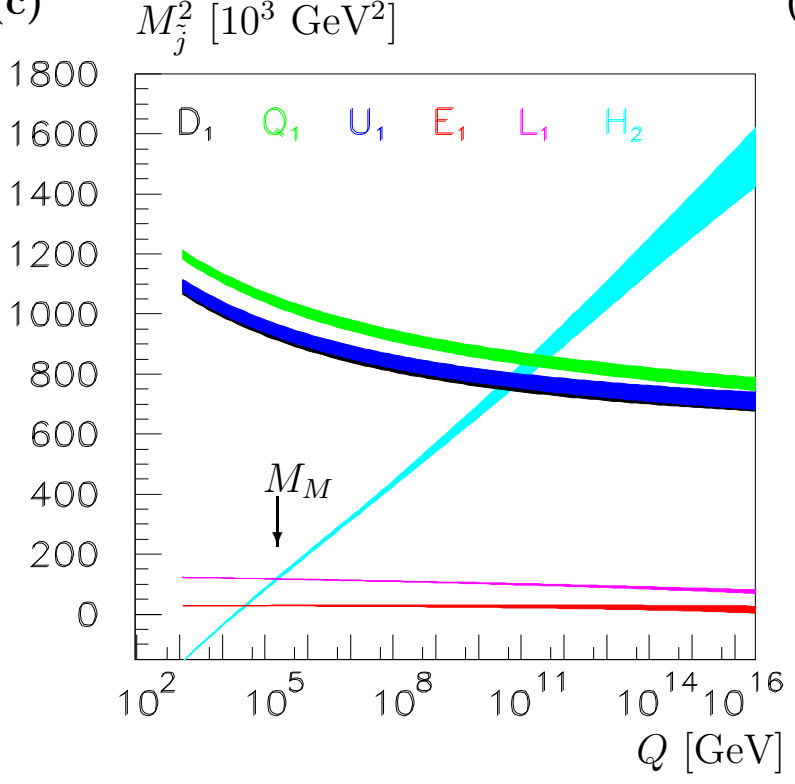

(b)

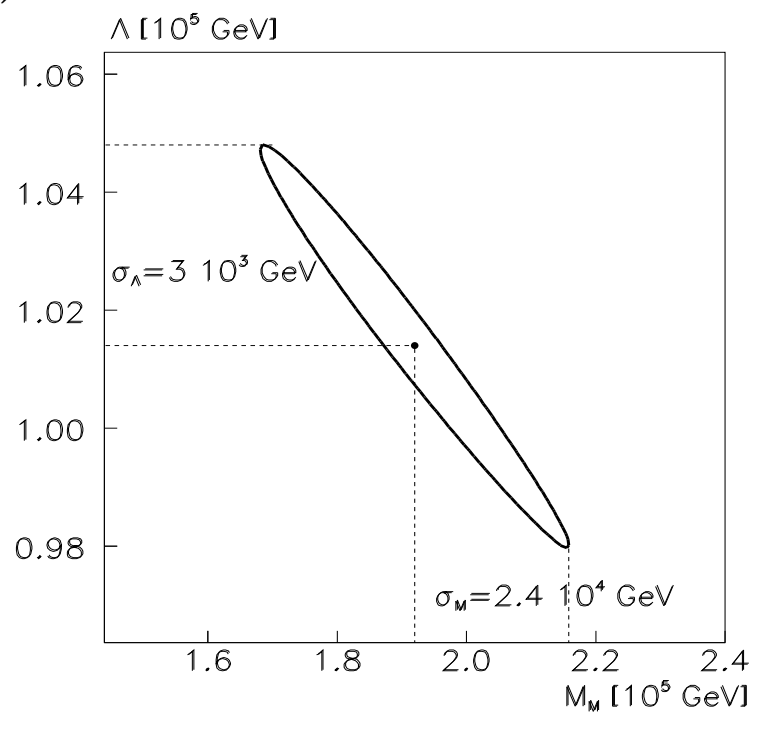

(d)

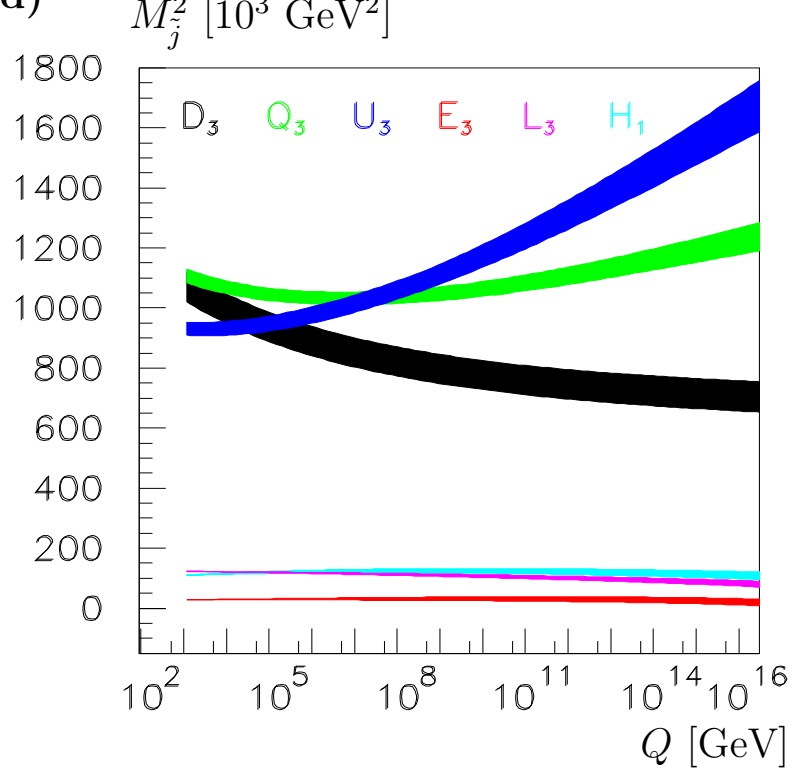

Figure 4: GMSB: Evolution of (a) gaugino mass parameters; (b) $\Lambda-M_{M}$ determination in the bottom-up approach. (c) first-generation sfermion mass parameters and Higgs mass parameter $M_{\mathrm{H}_{2}}^{2}$; (d) third-generation sfermion mass parameters and Higgs mass parameter $M_{H_{1}}^{2}$; The point probed, SPS\#8, is characterized by the parameters $M_{M}=200 \mathrm{TeV}, \Lambda=$ $100 \mathrm{TeV}, N_{5}=1, \tan \beta=15$, and $\operatorname{sign}(\mu)=(+)$. [The widths of the bands indicate the $1 \sigma$ CL.] 
are generated by loops of scalar and fermionic messenger component fields; $N_{i}$ is the multiplicity of messengers in the $5+\overline{5}$ and $10+\overline{10}$ vector-like multiplets, and

$$
g(x)=\frac{1+x}{x^{2}} \log (1+x)+(x \rightarrow-x)
$$

is the messenger-scale threshold function [62] which approaches unity for $\Lambda \ll M_{M}$. Masses of the scalar fields in the visible sector are generated by 2-loop effects of gauge/gaugino and messenger fields:

$$
M_{\tilde{j}}^{2}\left(M_{M}\right)=2\left(N_{5}+3 N_{10}\right) f\left(\Lambda / M_{M}\right) \sum_{i=1}^{3} k_{i} C_{i}^{j} \alpha_{i}^{2}\left(M_{M}\right) \Lambda^{2}
$$

with $k_{i}=1,1,3 / 5$ for $S U(3), S U(2)$, and $U(1)$, respectively; the coefficients $C_{i}^{j}$ are the quadratic Casimir invariants, being $4 / 3,3 / 4$, and $Y^{2} / 4$ for the fundamental representations $\tilde{j}$ in the groups $i=S U(3), S U(2)$ and $U(1)$, with $Y=2\left(Q-I_{3}\right)$ denoting the usual hypercharge; also the threshold function 62

$$
f(x)=\frac{1+x}{x^{2}}\left[\log (1+x)-2 \operatorname{Li}_{2}\left(\frac{x}{1+x}\right)+\frac{1}{2} \operatorname{Li}_{2}\left(\frac{2 x}{1+x}\right)\right]+(x \rightarrow-x)
$$

approaches unity for $\Lambda \ll M_{M}$. As evident from Eq.(21) scalar particles with identical Standard-Model charges squared have equal masses at the messenger scale $M_{M}$. In the minimal version of GMSB, the $A$ parameters are generated at 3-loop level and they are practically zero at $M_{M}$.

We have investigated this scheme for the point $\Lambda=100 \mathrm{TeV}, M_{M}=200 \mathrm{TeV}, N_{5}=1$, $N_{10}=0, \tan \beta=15$ and $\mu>0$ corresponding to the Snowmass Point SPS\#8. We find for the low energy data: $\operatorname{BR}(b \rightarrow s \gamma)=3.7 \cdot 10^{-4}, \Delta[g-2]_{\mu}=15 \cdot 10^{-10}, \Delta \rho=64 \cdot 10^{-5}$. The evolution of the gaugino and sfermion mass parameters of the first and third generation as well as the Higgs mass parameters, including 2-loop $\beta$-functions, are presented in Fig. 4 . Owing to the influence of the $A$-parameters in the 2-loop RGEs for the gaugino mass parameters, the gaugino mass parameters do not meet at the same point as the gauge couplings in this scheme. It is obvious from the figure that the GMSB scenario cannot be confused with the universal supergravity scenarid]. [Specific experimental signatures generated in the decays of the next to lightest supersymmetric particle, the neutralino $\tilde{\chi}_{1}^{0}$ or the stau $\tilde{\tau}_{1}$, to gravitinos which are very light in GMSB, provide a complementary experimental discriminant, see [17, 64]].

The bands of the slepton $L$-doublet mass parameter $M_{\tilde{L}}^{2}$ and the Higgs parameter $M_{H_{2}}^{2}$, which carry the same moduli of standard-model charges, cross at the scale $M_{M}$. The

\footnotetext{
${ }^{5}$ The same formulae as in Appendix B.1 apply for the GMSB boundary conditions at the electroweak scale by replacing $M_{U}$ by $M_{M}$, the GMSB scale.

${ }^{6} \mathrm{~A}$ comparison of the mass characteristics at the low scale between mSUGRA and GMSB in a top-down approach is presented in Ref. 63].
} 
Table 6: Average ratios of the scalar mass parameters as predicted in GMSB solely by group factors and gauge couplings.

\begin{tabular}{c||c|c} 
Mass $^{2}$ Ratios & $<$ GMSB $>$ & $\neq$ mSUGRA \\
\hline \hline$H_{2}^{2} / L_{1}^{2}$ & 1 & - \\
\hline$E_{1}^{2} / L_{1}^{2}$ & 0.25 & $\geq 0.8$ \\
$Q_{1}^{2} / L_{1}^{2}$ & 8.87 & $\leq 3.2$ \\
$U_{1}^{2} / L_{1}^{2}$ & 8.03 & $\leq 3.0$ \\
$D_{1}^{2} / L_{1}^{2}$ & 7.95 & $\leq 3.2$ \\
$H_{1}^{2} / L_{1}^{2}$ & 1 & $\leq 1.0$ \\
\hline
\end{tabular}

crossing, which is indicated by an arrow in Fig. [t(c), is a necessary condition (in the minimal form) for the GMSB scenario to be realized.

The two scales $\Lambda$ and $M_{M}$ can be extracted from the spectrum of the gaugino and scalar particles. Combining the two species allows to determine the multiplicity coefficient $\left(N_{5}+3 N_{10}\right)$ in addition. The dependence of the spectra on $\Lambda$ is, trivially, very strong. The messenger scale $M_{M}$ can be determined only from the running of the masses between the messenger scale and the electroweak scale ; despite being governed by weakly varying logarithms, the accuracy in determining $M_{M}$ is surprisingly good. For the point analyzed in the example above, the following accuracy for the mass parameters and the messenger multiplicity has been found:

$$
\begin{aligned}
\Lambda & =(1.01 \pm 0.03) \cdot 10^{2} \mathrm{TeV} \\
M_{M} & =(1.92 \pm 0.24) \cdot 10^{2} \mathrm{TeV} \\
N_{5}+3 N_{10} & =0.978 \pm 0.056
\end{aligned}
$$

The correlation between $\Lambda$ and $M_{M}$ is shown in Fig. 国(b).

While the gaugino masses in GMSB evolve nearly in the same way as in mSUGRA, the running of the scalar masses is quite different in both theories. Moreover, at the messenger scale the ratios of scalar masses squared in the simplest version of GMSB are determined solely by group factors and gauge couplings, being independent of the specific GMSB characteristics, i. e. messenger multiplicities and $\Lambda$ mass scale:

$$
M_{\tilde{j}}^{2}\left(M_{M}\right) / M_{\tilde{j}^{\prime}}^{2}\left(M_{M}\right)=\sum_{i=1}^{3} k_{i} C_{i}^{j} \alpha_{i}^{2}\left(M_{M}\right) / \sum_{i=1}^{3} k_{i} C_{i}^{j^{\prime}} \alpha_{i}^{2}\left(M_{M}\right) .
$$

The predictions for these ratios are listed in Table 6. The ratios in GMSB are distinctly different from the ratios in mSUGRA, taken at the scale where the upper boundary of the $2 \sigma$ band for $H_{2}^{2} / L_{1}^{2}$ approaches unity from below. [Ideally all ratios approach unity only at 
a)

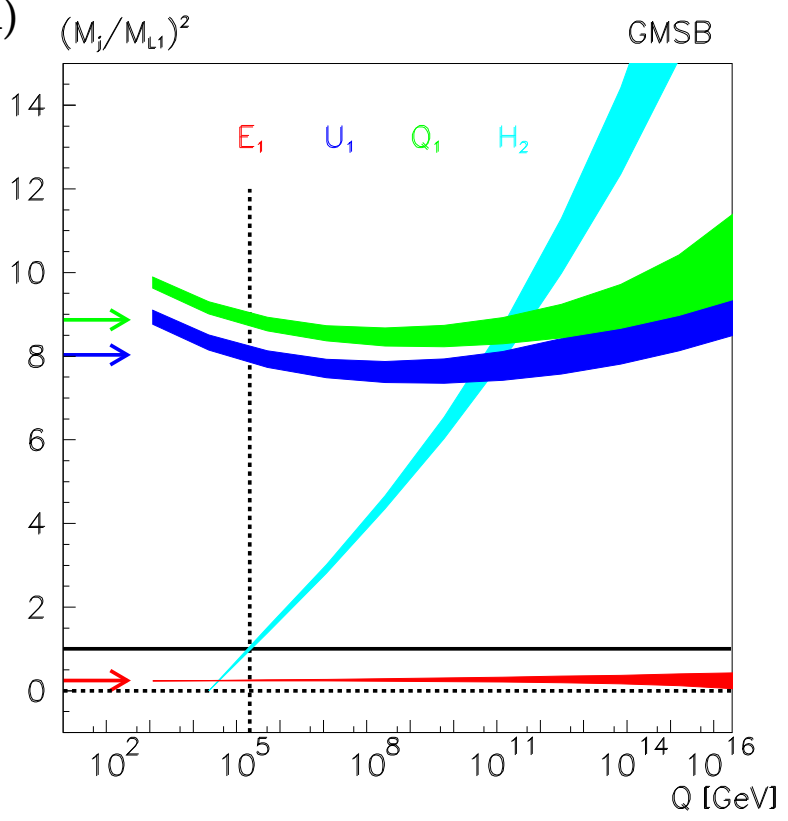

b)

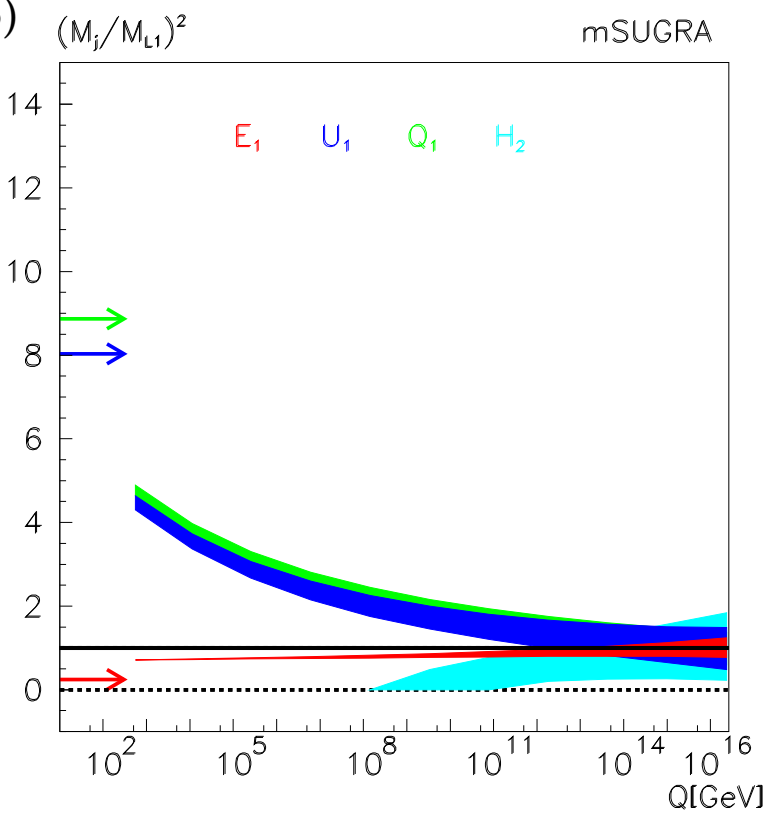

Figure 5: Evolution of representative ratios of first generation scalar masses squared, a) in case of GMSB; b) in case of mSUGRA. The messenger scale $M_{M}$ is defined as the scale where $M_{H_{2}}=M_{L_{1}}$. The arrows in both figures indicate the expectation values of the mass ratios squared in GMSB at the scale $M_{M}$.

the grand unification scale $M_{U}$ in mSUGRA.] The distinct differences between GMSB and mSUGRA are clearly visible in Figs. 5(a) vs. (b).

\section{$4 \quad$ String Induced Supersymmetry Breaking}

In the previously analyzed SUGRA and GMSB models the structure of the supersymmetry breaking mechanisms sui generis and the fields involved in the hidden sectors are shielded from the eigen-world. Four-dimensional strings naturally give rise to a minimal set of fields for inducing supersymmetry breaking; they play the rôle of the fields in the hidden sectors: the dilaton $S$ and the moduli $T_{m}$ chiral superfields which are generically present in large classes of 4-dimensional heterotic string theories']. The vacuum expectation values of $S$ and $T_{m}$, generated by genuinely non-perturbative effects, determine the soft supersymmetry breaking parameters. In this approach, grand unification at the standard scale can be reconciled with the higher string scale by moduli dependent string loop corrections.

In the following we assume that all moduli fields get the same vacuum expectation values and that they couple in the same way to matter fields. Therefore, we omit the index $m$ and take only one moduli field $T$. The properties of the supersymmetric theories are quite different for dilaton and moduli dominated scenarios. This can be quantified by introducing

\footnotetext{
${ }^{7}$ For other scenarios see Ref. [65].
} 
a mixing angle $\theta$, characterizing the $\tilde{S}$ and $\tilde{T}$ wave functions of the Goldstino, which is associated with the breaking of supersymmetry and which is absorbed to generate the mass of the gravitino:

$$
\tilde{G}=\sin \theta \tilde{S}+\cos \theta \tilde{T}
$$

The mass scale is set by the second parameter of the theory, the gravitino mass $m_{3 / 2}$.

A dilaton dominated scenario, i.e. $\sin \theta \rightarrow 1$, leads to universal boundary conditions of the soft supersymmetry breaking parameters. Universality is broken only slightly by small loop effects. On the other hand, in moduli field dominated scenarios, $\cos \theta \rightarrow 1$, the gaugino mass parameters are universal to lowest order [broken only in higher orders], but universality is not realized for the scalar mass parameters in general. The breaking is quantified by modular weights $n_{j}$ characterizing the couplings between the matter and the moduli fields in orbifold compactifications. Within one generation significant differences between left and right field components and between sleptons and squarks can occur; since these patterns are modified only by small loop effects between different generations, flavourchanging neutral effects remain suppressed.

In leading order, and next-to-leading order parameterized by the quantities $\Delta M$, the masses [13] are given by the following expressions for the gaugino sector,

$$
\begin{aligned}
M_{i}= & -g_{i}^{2} m_{3 / 2} s \sqrt{3} \sin \theta+\Delta M_{i} \\
\Delta M_{i}= & -g_{i}^{2} m_{3 / 2}\left\{b_{i}+s \sqrt{3} \sin \theta g_{s}^{2}\left(C_{i}-\sum_{j} C_{i}^{j}\right)\right. \\
& \left.+2 t \cos \theta G_{2}(t)\left[\delta_{\mathrm{GS}}+b_{i}-2 \sum_{j} C_{i}^{j}\left(1+n_{j}\right)\right]\right\} / 16 \pi^{2}
\end{aligned}
$$

and for the scalar sector,

$$
\begin{aligned}
M_{\tilde{j}}^{2}=m_{3 / 2}^{2} & \left(1+n_{j} \cos ^{2} \theta\right)+\Delta M_{\tilde{j}}^{2} \\
\Delta M_{\tilde{j}}^{2}=m_{3 / 2}^{2} & \left\{\gamma_{j}+2 t \cos \theta G_{2}(t) \sum_{k m} \gamma_{j}^{k m}\left(n_{j}+n_{k}+n_{m}+3\right)\right. \\
& \left.+2 \sqrt{3} s \sin \theta\left[\sum_{i} \gamma_{j}^{i} g_{i}^{2}-\frac{1}{2 s} \sum_{k m} \gamma_{j}^{k m}\right]\right\}
\end{aligned}
$$

while the $A$ parameters read

$$
\begin{aligned}
A_{j k m} & =-m_{3 / 2}\left[2 t \cos \theta\left(n_{j}+n_{k}+n_{m}+3\right) G_{2}(t)-\frac{\sin \theta}{\sqrt{3}}\right]+\Delta A_{j k m} \\
\Delta A_{j k m} & =m_{3 / 2}\left(\gamma_{j}+\gamma_{k}+\gamma_{m}\right)
\end{aligned}
$$

The mass $m_{3 / 2}$ is the gravitino mass introduced earlier. The gravitino mass can be expressed in terms of the Kähler potential $K$ and the superpotential $W$, which include the (nonperturbative) solutions of all the fields at the string scale: $m_{3 / 2}=\langle\exp (K / 2) \bar{W}\rangle \cdot s=\langle S\rangle$ is

\footnotetext{
${ }^{8}$ For other mechanisms of breaking universality see e.g. Ref. [66].
} 
the vacuum expectation values of the dilaton field. $t=\langle T\rangle / m_{3 / 2}$ is the vacuum expectation value of the moduli field(s), and $G_{2}(t)=2 \zeta(t)+1 / 2 t$ is the non-holomorphic Eisenstein function with $\zeta$ denoting the Riemann zeta function. $\delta_{G S}$ is the parameter of the GreenSchwarz counterterm. $\gamma_{j}$ are the anomalous dimensions of the matter fields, the $\gamma_{j}^{i}$ and $\gamma_{j}^{k m}$ are their gauge and Yukawa parts, respectively. $C_{i}, C_{i}^{j}$ are the quadratic Casimir operators for the gauge group $G_{i}$, respectively, in the adjoint representation and in the matter representation.

In the case of the gaugino mass parameters the next-to-leading order effects induce a splitting proportional to the beta functions $b_{i}$ which is large enough to be "measured" at future collider experiments as demonstrated in Fig. 6(a) and (b). In the case of the scalar mass parameters the next-to-leading order contributions generate small departures from non-universality between the generations even if the corresponding modular weight is generation independent. These departures are proportional to the Yukawa couplings squared so that the third generation, in particular the stop sector, is mainly affected.

Scenarios have been found in which the phenomenological unification of the three gauge couplings can be reconciled with a string mass scale which is an order of magnitude larger than the unification scale [67:

$$
\alpha_{i}^{-1}\left(M_{U}\right)=\alpha^{-1}\left(M_{\text {String }}\right)+\Delta \alpha_{i}^{-1}
$$

The corrections $\Delta \alpha_{i}^{-1}$ depend on the value of the moduli fields and the modular weights:

$$
\Delta \alpha_{i}^{-1}=\frac{1}{4 \pi}\left(b_{i}^{\prime}-b_{G S}\right) \log |\eta(t)|^{4}
$$

where $\eta(t)$ is the Dedekind $\eta$ function and

$$
\begin{aligned}
& b_{3}^{\prime}=9+\sum_{i=1}^{3}\left(2 n_{Q_{i}}+n_{U_{i}}+n_{D_{i}}\right) \\
& b_{2}^{\prime}=15+\sum_{i=1}^{3}\left(3 n_{Q_{i}}+n_{L_{i}}\right)+n_{H_{1}}+n_{H_{2}} \\
& b_{1}^{\prime}=\frac{99}{5}+\frac{1}{5} \sum_{i=1}^{3}\left(n_{Q_{i}}+8 n_{U_{i}}+2 n_{D_{i}}+3 n_{L_{i}}+6 n_{E_{i}}\right)+\frac{3}{5}\left(n_{H_{1}}+n_{H_{2}}\right)
\end{aligned}
$$

as compared to the one-loop $S U(3) \times S U(2) \times U(1) \beta$-functions $\left(b_{3}, b_{2}, b_{1}\right)=33 / 5,1,-3$.

We have analyzed a mixed dilaton/moduli superstring scenario but with dominating dilaton field component, $\sin ^{2} \theta=0.9$, and with different couplings of the moduli field to the $(\mathrm{L}, \mathrm{R})$ sleptons, the $(\mathrm{L}, \mathrm{R})$ squarks and to the Higgs fields, corresponding to O-I representation $n_{L_{i}}=-3, n_{E_{i}}=-1, n_{H_{1}}=n_{H_{2}}=-1, n_{Q_{i}}=0, n_{D_{i}}=1$ and $n_{U_{i}}=-2$, that is one out of several assignments that is adopted quite frequently in the literature. The gravitino mass is chosen to be $180 \mathrm{GeV}$ in this analysis. We find for the low energy data: $\mathrm{BR}(b \rightarrow s \gamma)=3.1 \cdot 10^{-4}, \Delta[g-2]_{\mu}=14 \cdot 10^{-10}, \Delta \rho=13 \cdot 10^{-5} ;$ and $\Omega h^{2}=0.25$. 
(a) $-1 / M_{i}\left[\mathrm{GeV}^{-1}\right] \times 10^{-2}$

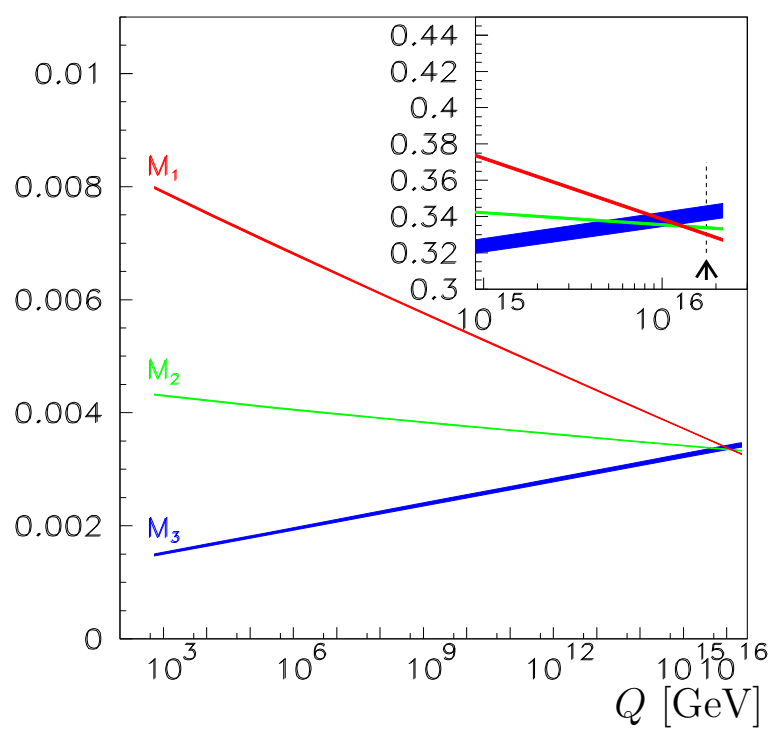

(b)

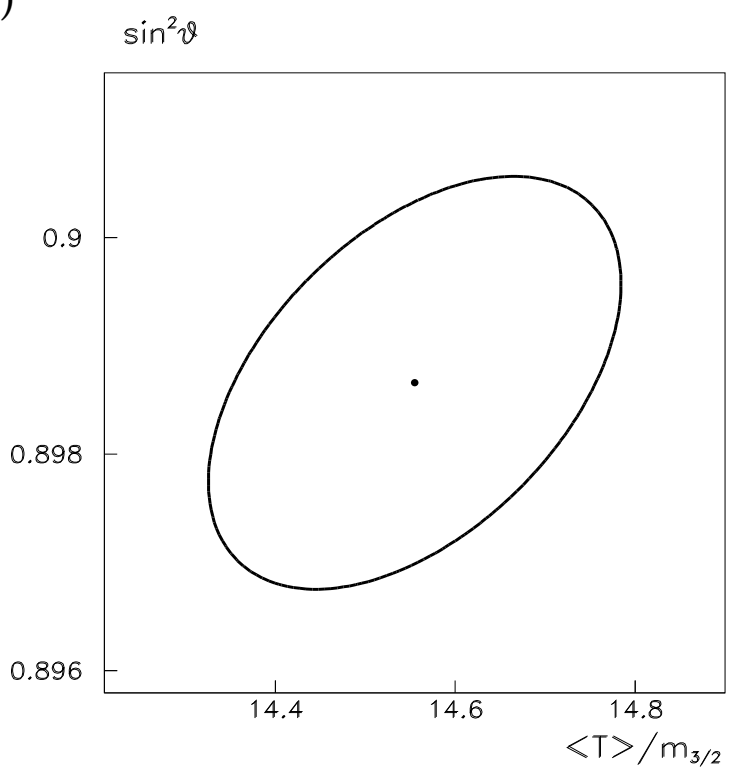

(d)

(c) $\quad M_{\tilde{j}}^{2}\left[10^{3} \mathrm{GeV}^{2}\right]$

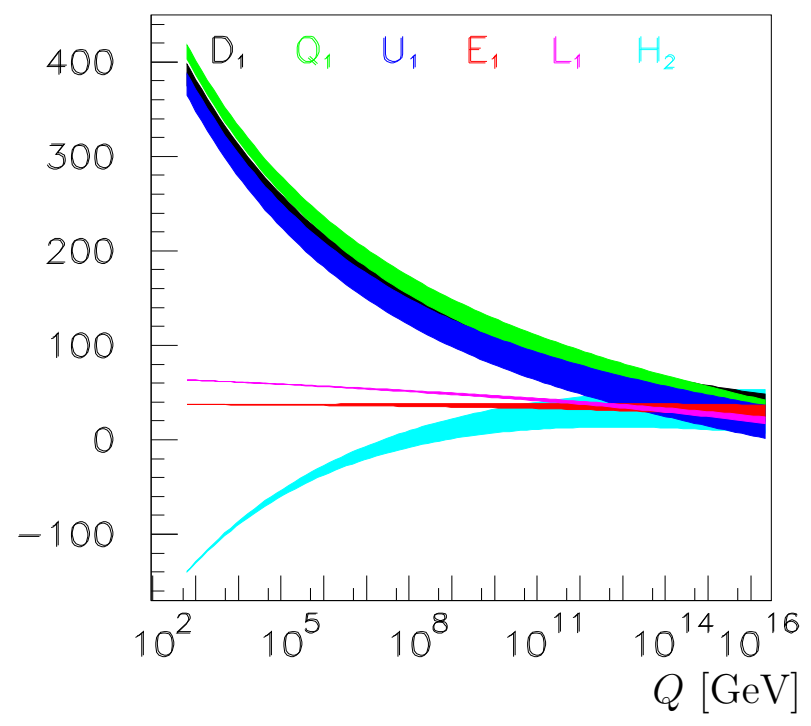

$M_{\tilde{j}}^{2}\left[10^{3} \mathrm{GeV}^{2}\right]$

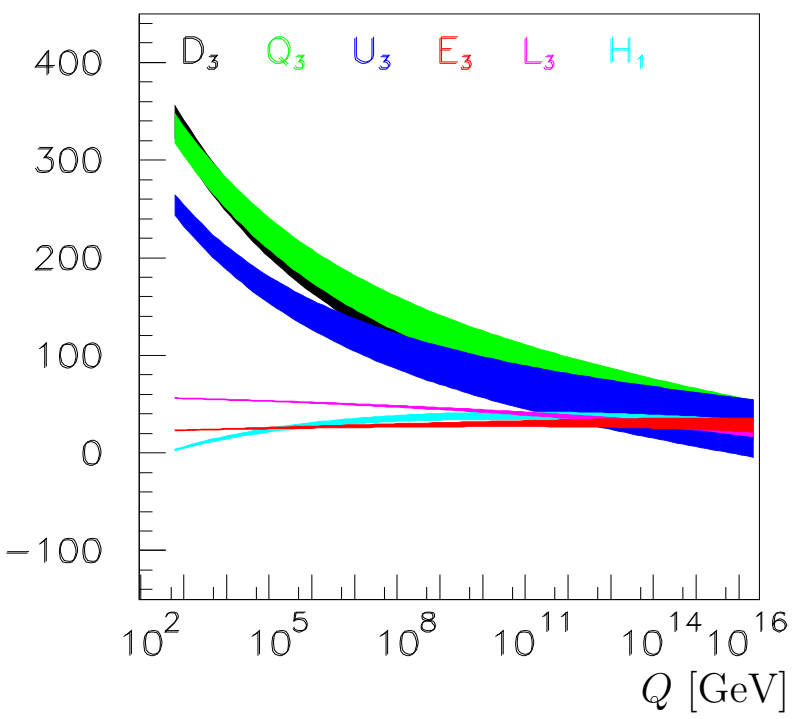

Figure 6: String Scenario: Evolution of (a) gaugino mass parameters [the insert expands on the breaking of universality at the GUT scale]; (b) correlation between the mixing parameter $\sin ^{2} \theta$ and the vacuum expectation value of the moduli field $\langle T\rangle$; (c) evolution of first-generation sfermion mass parameters and Higgs mass parameters $M_{H_{2}}^{2}$; (d) evolution of third generation sfermion mass parameters and Higgs mass parameters $M_{H_{1}}^{2}$. The point probed is characterized by the parameters $m_{3 / 2}=180 \mathrm{GeV}, \delta_{G S}=0, \sin ^{2} \theta=0.9$, $\langle T\rangle=14 m_{3 / 2}, \tan \beta=10, \operatorname{sign}(\mu)=(-), n_{L_{i}}=-3, n_{E_{i}}=-1, n_{Q_{i}}=0, n_{U_{i}}=-2, n_{D_{i}}=1$ and $n_{H_{1}}=n_{H_{2}}=-1$. [The widths of the bands indicate the $1 \sigma$ CL.] 
Table 7: Representative gaugino/scalar mass parameters and couplings as determined at the electroweak scale and evolved to the GUT scale in the string scenario; based on LHC and LC simulations. $M_{L_{1,3}}^{2}, M_{Q_{1,3}}^{2}$ are the slepton and squark isodoublet parameters of the first and third family whereas $M_{E_{1,3}}^{2}, M_{U_{1,3}}^{2}$ and $M_{D_{1,3}}^{2}$ are the the slepton and squark isosinglet parameters of the first and third family. [The errors quoted correspond to $1 \sigma$.

\begin{tabular}{|c|c|c|}
\hline & exp. input & GUT value \\
\hline$M_{1}[\mathrm{GeV}]$ & $-124.98 \pm 0.29$ & $-303.22 \pm 0.65$ \\
\hline$M_{2}[\mathrm{GeV}]$ & $-231.00 \pm 0.50$ & $-299.64 \pm 0.52$ \\
\hline$M_{3}[\mathrm{GeV}]$ & $-677.3 \pm 7.6$ & $-292.4 \pm 3.3$ \\
\hline$\mu$ & $-377.59 \pm 0.29$ & $-375.5 \pm 1.2$ \\
\hline$M_{L_{1}}^{2}\left[\mathrm{GeV}^{2}\right]$ & $(6.354 \pm 0.005) \cdot 10^{4}$ & $(2.17 \pm 0.43) \cdot 10^{4}$ \\
\hline$M_{E_{1}}^{2}\left[\mathrm{GeV}^{2}\right]$ & $(3.739 \pm 0.005) \cdot 10^{4}$ & $(2.88 \pm 0.86) \cdot 10^{4}$ \\
\hline$M_{Q_{1}}^{2}\left[\mathrm{GeV}^{2}\right]$ & $(4.16 \pm 0.09) \cdot 10^{5}$ & $(3.1 \pm 1.3) \cdot 10^{4}$ \\
\hline$M_{U_{1}}^{2}\left[\mathrm{GeV}^{2}\right]$ & $(3.80 \pm 0.12) \cdot 10^{5}$ & $(2.5 \pm 1.9) \cdot 10^{4}$ \\
\hline$M_{D_{1}}^{2}\left[\mathrm{GeV}^{2}\right]$ & $(3.88 \pm 0.13) \cdot 10^{5}$ & $(3.5 \pm 1.7) \cdot 10^{4}$ \\
\hline$M_{L_{3}}^{2}\left[\mathrm{GeV}^{2}\right]$ & $(5.635 \pm 0.039) \cdot 10^{4}$ & $(2.18 \pm 0.46) \cdot 10^{4}$ \\
\hline$M_{E_{3}}^{2}\left[\mathrm{GeV}^{2}\right]$ & $(2.253 \pm 0.024) \cdot 10^{4}$ & $(2.90 \pm 0.93) \cdot 10^{4}$ \\
\hline$M_{Q_{3}}^{2}\left[\mathrm{GeV}^{2}\right]$ & $(3.28 \pm 0.13) \cdot 10^{5}$ & $(3.2 \pm 2.1) \cdot 10^{4}$ \\
\hline$M_{U_{3}}^{2}\left[\mathrm{GeV}^{2}\right]$ & $(2.58 \pm 0.15) \cdot 10^{5}$ & $(2.6 \pm 3.3) \cdot 10^{4}$ \\
\hline$M_{D_{3}}^{2}\left[\mathrm{GeV}^{2}\right]$ & $(3.53 \pm 0.15) \cdot 10^{5}$ & $(3.5 \pm 1.8) \cdot 10^{4}$ \\
\hline$M_{H_{1}}^{2}\left[\mathrm{GeV}^{2}\right]$ & $(3.80 \pm 0.82) \cdot 10^{3}$ & $(2.85 \pm 0.62) \cdot 10^{4}$ \\
\hline$M_{H_{2}}^{2}\left[\mathrm{GeV}^{2}\right]$ & $(-1.429 \pm 0.004) \cdot 10^{5}$ & $(3.1 \pm 2.7) \cdot 10^{4}$ \\
\hline$A_{t}[\mathrm{GeV}]$ & $452 \pm 17$ & $-96 \pm 64$ \\
\hline $\tan \beta$ & $9.93 \pm 0.88$ & - \\
\hline
\end{tabular}

Given this set of superstring induced parameters, the evolution of the gaugino and scalar mass parameters is displayed in Fig. 6. The pattern of the trajectories is remarkably different from other scenarios. The breaking of universality in the gaugino sector, induced by string threshold corrections, is pronounced, cf. Table 7 .

In fact, the differences can be exploited to determine superstring parameters as argued above. The number of observables in the set of gauge couplings $g_{a}$, gaugino masses $M_{a}$ and scalar masses $M_{\tilde{j}}$ exceeds the number of parameters in the superstring effective field theory: the gravitino mass $m_{3 / 2}$, the dilaton/moduli mixing angle $\sin \theta$, the ground-state value of the moduli field $\langle T\rangle$ and the ground-state value of the dilaton field $\langle S\rangle$. The latter is at tree-level directly related to the string coupling: $1 / g_{s}^{2}=\langle S\rangle$.

Based on the "experimental" input observables, the fundamental parameters of the string effective field theory can be reconstructed; the reconstructed values are compared with the 
Table 8: Comparison of the experimentally reconstructed values with the ideal fundamental parameters in a specific example for a string effective field theory.

\begin{tabular}{c||c|rll} 
Parameter & Ideal & \multicolumn{2}{|c}{ Reconstructed } \\
\hline \hline$m_{3 / 2}$ & 180 & 179.9 & \pm & 0.4 \\
$\langle S\rangle$ & 2 & 1.998 & \pm & 0.006 \\
$\langle T\rangle$ & 14 & 14.6 & \pm & 0.2 \\
$\sin ^{2} \theta$ & 0.9 & 0.899 & \pm & 0.002 \\
$g_{s}^{2}$ & 0.5 & 0.501 & \pm & 0.002 \\
$\delta_{G S}$ & 0 & 0.1 & \pm & 0.4 \\
\hline$n_{L}$ & -3 & -2.94 & \pm & 0.04 \\
$n_{E}$ & -1 & -1.00 & \pm & 0.05 \\
$n_{Q}$ & 0 & 0.02 & \pm & 0.02 \\
$n_{U}$ & -2 & -2.01 & \pm & 0.02 \\
$n_{D}$ & +1 & 0.80 & \pm & 0.04 \\
$n_{H_{1}}$ & -1 & -0.96 & \pm & 0.06 \\
$n_{H_{2}}$ & -1 & -1.00 & \pm & 0.02 \\
\hline $\tan \beta$ & 10 & 10.00 & \pm & 0.13
\end{tabular}

ideal values in Table 8. The errors for the basic parameters $\sin \theta,\langle T\rangle / m_{3 / 2}$ are displayed in Figs. 6(b).

Thus, high-precision measurements at high energy proton and $e^{+} e^{-}$linear colliders provide access to crucial derivative parameters in string theories.

\section{Conclusions}

In supersymmetric theories stable extrapolations can be performed from the electroweak scale to the Grand Unification scale close to the Planck scale. This feature has been compellingly demonstrated in the evolution of the three gauge couplings to the unification point in the minimal supersymmetric theory.

Such extrapolations are made possible by high-precision measurements of the low-energy parameters. The operation of the $e^{+} e^{-}$colliders LEP and SLC has been crucial in this context. In the near future an enormous extension of this area will be possible if measurements at LHC and prospective $e^{+} e^{-}$linear colliders are combined to draw, if realized in Nature, a comprehensive high-precision picture of the supersymmetric particles and their interactions. Based merely on measurements at low energies, the parameters of the theory can be evolved to high scales by means of renormalization group techniques.

Supersymmetric theories and their breaking mechanisms have the simplest structures and 
the greatest regularities at high scales. Extrapolations to high scales are therefore crucial to uncover the regularities. The bottom-up approach in the extrapolation of parameters measured at low scales to the high scales provides the most transparent picture. In this way the basis of the SUSY breaking mechanism can be explored and the crucial elements of the fundamental supersymmetric theory can be reconstructed. The method can thus be used to explore particle physics phenomena at a scale where, eventually, particle physics is linked to gravity.

Apart from other examples, we have focused on two interesting scenarios in this approach. The universality of gaugino and scalar mass parameters in minimal supergravity can be demonstrated very clearly if realized in the supersymmetric theory. Small deviations from universality, on the other hand, may be exploited to measure the fundamental parameters in superstring effective field theories, i.e. the strength of dilaton and moduli fields, their mixing and the modular weights. In this way, high-precision extrapolations of gauge and supersymmetric parameters can establish direct contact between superstring theory and experiment.

Many more refinements of the theoretical calculations and future experimental analyses will be necessary to expand the picture we have drawn in this first attempt. However, the prospect of exploring elements of the ultimate unification of the interactions provides a strong impetus to this direction.

\section{Acknowledgements}

We are very grateful for enlightening discussions with J. Kalinowski, G.L. Kane, B.D. Nelson and H.P. Nilles. G.A.B. is grateful for the hospitality extended to him in long-term visits to CERN and DESY, for support from the British Council ARC programme and Intas grant 00-00679. W.P. is supported by the Erwin Schrödinger fellowship No. J2095 of the 'Fonds zur Förderung der wissenschaftlichen Forschung' of Austria FWF and partly by the Swiss 'Nationalfonds'.

\section{A One-loop RGEs}

In this first appendix we collect the one-loop renormalization group equations (RGEs) including right-handed neutrinos.

Using the notation for the gauge and Yukawa couplings

$$
\alpha_{i}=\frac{g_{i}^{2}}{16 \pi^{2}}, i=1,2,3 ; \quad Y_{k}=\frac{y_{k}^{2}}{16 \pi^{2}}, k=t, b, \tau, \nu
$$

the one-loop RG equations can be written as

$$
\dot{\alpha}_{i}=-b_{i} \alpha_{i}^{2}
$$




$$
\dot{Y}_{k}=Y_{k}\left(\sum_{i} c_{k i} \alpha_{i}-\sum_{l} a_{k l} Y_{l}\right)
$$

where the dot denotes the derivative with respect to $t=\log M_{G U T}^{2} / Q^{2}$, and

$$
\begin{aligned}
b_{i} & =\{33 / 5,1,-3\}, \\
c_{t i} & =\{13 / 15,3,16 / 3\}, \quad c_{b i}=\{7 / 15,3,16 / 3\} \\
c_{\tau i} & =\{9 / 5,3,0\}, \quad c_{\nu i}=\{3 / 5,3,0\} \\
a_{t l} & =\{6,1,0,1\}, \quad a_{b l}=\{1,6,1,0\} \\
a_{\tau l} & =\{0,3,4,1\}, \quad a_{\nu l}=\{3,0,1,4\}
\end{aligned}
$$

while the RGEs for the gaugino mass parameters and the $A$-parameters read

$$
\begin{aligned}
\dot{M}_{i} & =-b_{i} \alpha_{i} M_{i}, \\
\dot{A}_{k} & =\sum_{i} c_{k i} \alpha_{i} M_{i}-\sum_{l} a_{k l} A_{l} .
\end{aligned}
$$

The RGEs for the soft SUSY breaking mass parameters of the third generation and the Higgs mass parameters are given by:

$$
\begin{aligned}
& \dot{M}_{L_{3}}=-2 Y_{\tau} X_{\tau}-2 Y_{\nu} X_{\nu}+\frac{6}{5} \alpha_{1} M_{1}^{2}+6 \alpha_{2} M_{2}^{2}+\frac{3}{5} S \\
& \dot{M}_{\nu_{R 3}}=-4 Y_{\nu} X_{\nu} \\
& \dot{M}_{E_{3}}=-4 Y_{\tau} X_{\tau}+\frac{24}{5} \alpha_{1} M_{1}^{2}-\frac{6}{5} S \\
& \dot{M}_{Q_{3}}=-2 Y_{b} X_{b}-2 Y_{t} X_{t}+\frac{2}{15} \alpha_{1} M_{1}^{2}+6 \alpha_{2} M_{2}^{2}+\frac{16}{3} \alpha_{3} M_{3}^{2}-\frac{1}{5} S, \\
& \dot{M}_{U_{3}}=-4 Y_{t} X_{t}+\frac{32}{15} \alpha_{1} M_{1}^{2}+\frac{16}{3} \alpha_{3} M_{3}^{2}+\frac{4}{5} S, \\
& \dot{M}_{D_{3}}=-4 Y_{b} X_{b}+\frac{8}{15} \alpha_{1} M_{1}^{2}+\frac{16}{3} \alpha_{3} M_{3}^{2}-\frac{2}{5} S, \\
& \dot{M}_{H_{1}}=-6 Y_{b} X_{b}-2 Y_{\tau} X_{\tau}+\frac{6}{5} \alpha_{1} M_{1}^{2}+6 \alpha_{2} M_{2}^{2}+\frac{3}{5} S, \\
& \dot{M}_{H_{2}}=-6 Y_{t} X_{t}-2 Y_{\nu} X_{\nu}+\frac{6}{5} \alpha_{1} M_{1}^{2}+6 \alpha_{2} M_{2}^{2}-\frac{3}{5} S,
\end{aligned}
$$

with

$$
\begin{aligned}
X_{t} & =M_{Q_{3}}^{2}+M_{U_{3}}^{2}+M_{H_{2}}^{2}+A_{t}^{2}, \\
X_{b} & =M_{Q_{3}}^{2}+m_{D}^{2}+M_{H_{1}}^{2}+A_{b}^{2}, \\
X_{\tau} & =M_{L_{3}}^{2}+M_{E_{3}}^{2}+M_{H_{1}}^{2}+A_{\tau}^{2}, \\
X_{\nu} & =M_{L_{3}}^{2}+M_{\nu_{R 3}}^{2}+M_{H_{2}}^{2}+A_{\nu}^{2}, \\
S & =M_{H_{2}}^{2}-M_{H_{2}}^{2}+\sum_{i=1}^{3}\left(M_{Q_{i}}^{2}-M_{L_{i}}^{2}-2 M_{U_{i}}^{2}+M_{D_{i}}^{2}+M_{E_{i}}^{2}\right) .
\end{aligned}
$$

The evolution equations for the first two generations are obtained by replacing appropriately the corresponding parameters and Yukawa couplings. 


\section{B Solutions of the one-loop RGEs}

In the following subsections we present the analytical solutions to the 1-loop RGEs including Yukawa couplings using the procedure of Ref. 68. We also include the generic trace term $S$ (see Eq.661)) in the solutions which had been neglected in Ref. [68]. In this appendix we mark all quantities defined at the the GUT-scale $M_{G U T}$ with a subscript G.

\section{B.1 mSUGRA Boundary Conditions at the GUT-scale}

The solutions for the case of the MSSM are summarized first for proper reference. The solution for the gauge couplings and Yukawa couplings are given by:

$$
\begin{aligned}
\alpha_{i}(t) & =\frac{\alpha_{i, G}}{1+b_{i} \alpha_{i, G} t} \\
Y_{k}(t) & =\frac{Y_{k, G} u_{k}}{1+a_{k k} Y_{k, G} \int_{0}^{t} u_{k}},
\end{aligned}
$$

where the functions $u_{k}$ obey the integral system of equations

$$
\begin{aligned}
u_{t} & =\frac{E_{t}}{\left(1+6 Y_{b, G} \int_{0}^{t} u_{b}\right)^{1 / 6}}, \\
u_{b} & =\frac{E_{b}}{\left(1+6 Y_{t, G} \int_{0}^{t} u_{t}\right)^{1 / 6}\left(1+4 Y_{\tau, G} \int_{0}^{t} u_{\tau}\right)^{1 / 4}}, \\
u_{\tau} & =\frac{E_{\tau}}{\left(1+6 Y_{b, G} \int_{0}^{t} u_{b}\right)^{1 / 2}},
\end{aligned}
$$

and the functions $E_{k}$ denote the products

$$
E_{k}=\prod_{i=1}^{3}\left(1+b_{i} \alpha_{i, G} t\right)^{c_{k i} / b_{i}} .
$$

The system of integral equations can be solved iteratively and a discussion on the convergence can be found in Ref. 68.

The gaugino mass parameters and the $A_{k}$ parameters are given by

$$
\begin{aligned}
M_{i}(t) & =\frac{M_{i, G}}{1+b_{i} \alpha_{i, G} t}=\frac{\alpha_{i}(t)}{\alpha_{i, G}} M_{i, G}, \\
A_{k} & =-e_{k}+\frac{A_{k, G} / Y_{k, G}+a_{k k} \int u_{k} e_{k}}{1 / Y_{k, G}+a_{k k} \int u_{k}},
\end{aligned}
$$

with the coefficients

$$
\begin{aligned}
e_{t} & =\tilde{F}_{t}+\frac{A_{b, G} \int u_{b}-\int u_{b} e_{b}}{1 / Y_{b, G}+6 \int u_{b}} \\
e_{b} & =\tilde{F}_{b}+\frac{A_{t, G} \int u_{t}-\int u_{t} e_{t}}{1 / Y_{t, G}+6 \int u_{t}}+\frac{A_{\tau, G} \int u_{\tau}-\int u_{\tau} e_{\tau}}{1 / Y_{\tau, G}+4 \int u_{\tau}} \\
e_{\tau} & =\tilde{F}_{\tau}+3 \frac{A_{b, G} \int u_{b}-\int u_{b} e_{b}}{1 / Y_{b, G}+6 \int u_{b}} \\
\tilde{F}_{k} & =t \sum_{i=1}^{3} c_{k i} M_{i, G} \alpha_{i}(t)
\end{aligned}
$$


The mass parameters of the first two generations $(k=1,2)$ can be expressed as

$$
\begin{aligned}
& M_{L_{k}}^{2}(t)=M_{L_{k}, G}^{2}+\frac{3}{2} f_{2}(t)+\frac{3}{10} f_{1}(t)+\frac{3}{5} S^{\prime}(t) \\
& M_{E_{k}}^{2}(t)=M_{E_{k}, G}^{2}+\frac{6}{5} f_{1}(t)-\frac{6}{5} S^{\prime}(t) \\
& M_{Q_{k}}^{2}(t)=M_{Q_{k}, G}^{2}+\frac{8}{3} f_{3}(t)+\frac{3}{2} f_{2}(t)+\frac{1}{30} f_{1}(t)-\frac{1}{5} S^{\prime}(t) \\
& M_{U_{k}}^{2}(t)=M_{U_{k}, G}^{2}+\frac{8}{3} f_{3}(t)+\frac{8}{15} f_{1}(t)+\frac{4}{5} S^{\prime}(t) \\
& M_{D_{k}}^{2}(t)=M_{D_{k}, G}^{2}+\frac{8}{3} f_{3}(t)+\frac{2}{15} f_{1}(t)-\frac{2}{5} S^{\prime}(t)
\end{aligned}
$$

with

$$
\begin{aligned}
f_{i}(t) & =\frac{M_{i, G}^{2}}{b_{i}}\left(1-\frac{1}{\left(1+\alpha_{i, G} b_{i} t\right)^{2}}\right) \\
S^{\prime}(t) & =\frac{1}{2 b_{1}}\left(S(t)-S\left(M_{U}\right)\right) \\
S(t) & =S\left(M_{U}\right)\left(1+\beta_{1} t\right)^{2} \\
S\left(M_{U}\right) & =M_{H_{1}, G}^{2}-M_{H_{2}, G}^{2}+\sum_{i=1}^{3}\left(M_{Q_{i}, G}^{2}-M_{L_{i}, G}^{2}-2 M_{U_{i}, G}^{2}+M_{D_{i}, G}^{2}+M_{E_{i}, G}^{2}\right),
\end{aligned}
$$

in agreement with Ref. [69]. The mass parameters for the third generation and the Higgs mass parameters are involved owing to the Yukawa couplings:

$$
\begin{aligned}
& M_{L_{3}}^{2}=M_{L_{3}, G}^{2}+\frac{80 f_{3}+123 f_{2}-103 / 5 f_{1}}{122}-\frac{3}{5} S^{\prime}(t)+\frac{3 \Delta \Sigma_{t}-18 \Delta \Sigma_{b}+35 \Delta \Sigma_{\tau}}{122}, \\
& M_{E_{3}}^{2}=M_{E_{3}, G}^{2}+\frac{80 f_{3}-60 f_{2}+16 f_{1}}{61}+\frac{6}{5} S^{\prime}(t)+\frac{3 \Delta \Sigma_{t}-18 \Delta \Sigma_{b}+35 \Delta \Sigma_{\tau}}{61}, \\
& M_{Q_{3}}^{2}=M_{Q_{3}, G}^{2}+\frac{128 f_{3}+87 f_{2}-11 f_{1}}{122}+\frac{1}{5} S^{\prime}(t)+\frac{17 \Delta \Sigma_{t}+20 \Delta \Sigma_{b}-5 \Delta \Sigma_{\tau}}{122} \\
& M_{U_{3}}^{2}=M_{U_{3}, G}^{2}+\frac{72 f_{3}-54 f_{2}+72 / 5 f_{1}}{61}-\frac{4}{5} S^{\prime}(t)+\frac{21 \Delta \Sigma_{t}-4 \Delta \Sigma_{b}+\Delta \Sigma_{\tau}}{61} \\
& M_{D_{3}}^{2}=M_{D_{3}, G}^{2}+\frac{56 f_{3}-42 f_{2}+56 / 5 f_{1}}{61}+\frac{2}{5} S^{\prime}(t)+\frac{-4 \Delta \Sigma_{t}+24 \Delta \Sigma_{b}-6 \Delta \Sigma_{\tau}}{61}, \\
& M_{H_{1}}^{2}=M_{H_{1}, G}^{2}+\frac{-240 f_{3}-3 f_{2}-57 / 5 f_{1}}{122}-\frac{3}{5} S^{\prime}(t)+\frac{-9 \Delta \Sigma_{t}+54 \Delta \Sigma_{b}+17 \Delta \Sigma_{\tau}}{122}, \\
& M_{H_{2}}^{2}=M_{H_{2}, G}^{2}+\frac{-272 f_{3}+21 f_{2}-89 / 5 f_{1}}{122}+\frac{3}{5} S^{\prime}(t)+\frac{63 \Delta \Sigma_{t}-12 \Delta \Sigma_{b}+3 \Delta \Sigma_{\tau}}{122},
\end{aligned}
$$

with

$$
\begin{aligned}
\Delta \Sigma_{k} & =\Sigma_{k}(t)-\Sigma_{k, G}, \\
\Sigma_{t} & =M_{Q_{3}}^{2}+M_{U_{3}}^{2}+M_{H_{2}}^{2}, \\
\Sigma_{b} & =M_{Q_{3}}^{2}+M_{D_{3}}^{2}+M_{H 1}^{2}, \\
\Sigma_{\tau} & =M_{L_{3}}^{2}+M_{E_{3}}^{2}+M_{H 1}^{2} .
\end{aligned}
$$


The explicit solution for $\Sigma_{k}$ read as:

$$
\Sigma_{k}=\xi_{k}+A_{k}^{2}+2 e_{k} A_{k}-\frac{A_{k, G}^{2} / Y_{k, G}-\Sigma_{k, G} / Y_{k, G}+a_{k k} \int u_{k} \xi_{k}}{1 / Y_{k, G}+a_{k k} \int u_{k}},
$$

with

$$
\begin{aligned}
\xi_{t}= & \tilde{E}_{t}+2 \tilde{F}_{t} \frac{A_{b, G} \int u_{b}-\int u_{b} e_{b}}{1 / Y_{b, G}+6 \int u_{b}}+7\left(\frac{A_{b, G} \int u_{b}-\int u_{b} e_{b}}{1 / Y_{b, G}+6 \int u_{b}}\right)^{2} \\
- & \frac{\left(\Sigma_{b, G}+A_{b, G}^{2}\right) \int u_{b}-2 A_{b, G} \int u_{b} e_{b}+\int u_{b} \xi_{b}}{1 / Y_{b, G}+6 \int u_{b}} \\
\xi_{b}= & \tilde{E}_{b}+2 \tilde{F}_{b}\left[\frac{A_{t, G} \int u_{t}-\int u_{t} e_{t}}{1 / Y_{t, G}+6 \int u_{t}}+\frac{A_{\tau, G} \int u_{\tau}-\int u_{\tau} e_{\tau}}{1 / Y_{\tau, G}+4 \int u_{\tau}}\right] \\
+ & 7\left(\frac{A_{t, G} \int u_{t}-\int u_{t} e_{t}}{1 / Y_{t, G}+6 \int u_{t}}\right)^{2}+5\left(\frac{A_{\tau, G} \int u_{\tau}-\int u_{\tau} e_{\tau}}{1 / Y_{\tau, G}+4 \int u_{\tau}}\right)^{2} \\
+ & 2\left(\frac{A_{t, G} \int u_{t}-\int u_{t} e_{t}}{1 / Y_{t, G}+6 \int u_{t}}\right)\left(\frac{A_{\tau, G} \int u_{\tau}-\int u_{\tau} e_{\tau}}{1 / Y_{\tau, G}+4 \int u_{\tau}}\right)-\frac{\left(\Sigma_{t, G}+A_{t, G}^{2}\right) \int u_{t}-2 A_{t, G} \int u_{t} e_{t}+\int u_{t} \xi_{t}}{1 / Y_{t, G}+4 \int u_{t}} \\
& -\frac{\left(\Sigma_{\tau, G}+A_{\tau, G}^{2}\right) \int u_{\tau}-2 A_{\tau, G} \int u_{\tau} e_{\tau}+\int u_{\tau} \xi_{\tau}}{1 / Y_{\tau}} \\
\xi_{\tau}= & \tilde{E}_{\tau}+6 \tilde{F}_{\tau} \frac{A_{b, G} \int u_{b}-\int u_{b} e_{b}}{1 / Y_{b, G}+6 \int u_{b}}+27\left(\frac{A_{b, G} \int u_{b}-\int u_{b} e_{b}}{1 / Y_{b, G}+6 \int u_{b}}\right)^{2} \\
- & 3 \frac{\left(\Sigma_{b, G}+A_{b, G}^{2}\right) \int u_{b}-2 A_{b, G} \int u_{b} e_{b}+\int u_{b} \xi_{b}}{1 / Y_{b, G}+6 \int u_{b}} \\
\tilde{E}_{k}= & t^{2}\left(\sum_{i=1}^{3} c_{k i} \alpha_{i} M_{i, G}\right)^{2}+2 t \sum_{i=1}^{3} c_{k i} \alpha_{i} M_{i, G}^{2}-t^{2} \sum_{i=1}^{3} c_{k i} b_{i} \alpha_{i}^{2} M_{i, G}^{2} \cdot
\end{aligned}
$$

Finally we express $t_{Z}=\log \left(M_{G U T}^{2} / m_{Z}^{2}\right)$ and $\alpha_{G}$ in terms of observables at the electroweak scale, using Eq.(62), by

$$
t_{Z}=\frac{4 \pi}{\left(b_{1}-b_{2}\right) \alpha\left(m_{Z}\right)}\left(\frac{3 \cos ^{2} \vartheta_{W}}{5}-\sin ^{2} \vartheta_{W}\right)
$$

and similarly for the gauge coupling at the GUT scale:

$$
\alpha_{G}=\frac{5 \alpha\left(m_{Z}\right)}{3} \frac{b_{1}-b_{2}}{\frac{5}{3} b_{1} \sin ^{2} \Theta_{W}-b_{2} \cos ^{2} \Theta_{W}}
$$

\section{B.2 Universal SUGRA Boundary Conditions at the GUT-scale including Right-handed Neutrinos}

Those formulae are noted in this subsection which are changed compared to the previous section in the range between $M_{U}$ and $M_{\nu_{R}}$. Below $M_{\nu_{R}}$, these quantities have the same

form as given above. In addition we note also the equations related to the right-handed 
neutrinos,

$$
\begin{aligned}
& u_{t}=\frac{E_{t}}{\left(1+6 Y_{b, G} \int_{0}^{t} u_{b}\right)^{1 / 6}\left(1+4 Y_{\nu, G} \int_{0}^{t} u_{\nu}\right)^{1 / 4}}, \\
& u_{\tau}=\frac{E_{\tau}}{\left(1+6 Y_{b, G} \int_{0}^{t} u_{b}\right)^{1 / 2}\left(1+4 Y_{\nu, G} \int_{0}^{t} u_{\nu}\right)^{1 / 4}}, \\
& u_{\nu}=\frac{E_{\nu}}{\left(1+6 Y_{t, G} \int_{0}^{t} u_{t}\right)^{1 / 2}\left(1+4 Y_{\tau, G} \int_{0}^{t} u_{\tau}\right)^{1 / 4}}, \\
& e_{t}=\tilde{F}_{t}+\frac{A_{b, G} \int u_{b}-\int u_{b} e_{b}}{1 / Y_{b, G}+6 \int u_{b}}+\frac{A_{\nu, G} \int u_{\nu}-\int u_{\nu} e_{\nu}}{1 / Y_{\nu, G}+4 \int u_{\nu}} \\
& e_{\tau}=\tilde{F}_{\tau}+3 \frac{A_{b, G} \int u_{b}-\int u_{b} e_{b}}{1 / Y_{b, G}+6 \int u_{b}}+\frac{A_{\nu, G} \int u_{\nu}-\int u_{\nu} e_{\nu}}{1 / Y_{\nu, G}+4 \int u_{\nu}}, \\
& e_{\nu}=\tilde{F}_{\nu}+3 \frac{A_{t, G} \int u_{t}-\int u_{t} e_{t}}{1 / Y_{t, G}+6 \int u_{t}}+\frac{A_{\tau, G} \int u_{\tau}-\int u_{\tau} e_{\tau}}{1 / Y_{\tau, G}+4 \int u_{\tau}} . \\
& \xi_{t}=\tilde{E}_{t}+2 \tilde{F}_{t}\left(\frac{A_{b, G} \int u_{b}-\int u_{b} e_{b}}{1 / Y_{b, G}+6 \int u_{b}}+\frac{A_{\nu, G} \int u_{\nu}-\int u_{\nu} e_{\nu}}{1 / Y_{\nu, G}+4 \int u_{\nu}}\right)+7\left(\frac{A_{b, G} \int u_{b}-\int u_{b} e_{b}}{1 / Y_{b, G}+6 \int u_{b}}\right)^{2} \\
& +5\left(\frac{A_{\nu, G} \int u_{\nu}-\int u_{\nu} e_{\nu}}{1 / Y_{\nu, G}+4 \int u_{\nu}}\right)^{2}+2\left(\frac{A_{b, G} \int u_{b}-\int u_{b} e_{b}}{1 / Y_{b, G}+6 \int u_{b}}\right)\left(\frac{A_{\nu, G} \int u_{\nu}-\int u_{\nu} e_{\nu}}{1 / Y_{\nu, G}+4 \int u_{\nu}}\right) \\
& -\frac{\left(\Sigma_{b, G}+A_{b, G}^{2}\right) \int u_{b}-2 A_{b, G} \int u_{b} e_{b}+\int u_{b} \xi_{b}}{1 / Y_{b, G}+6 \int u_{b}} \\
& -\frac{\left(\Sigma_{\nu, G}+A_{\nu, G}^{2}\right) \int u_{\nu}-2 A_{\nu, G} \int u_{\nu} e_{\nu}+\int u_{\nu} \xi_{\nu}}{1 / Y_{\nu, G}+4 \int u_{\nu}} \\
& \xi_{\tau}=\tilde{E}_{\tau}+2 \tilde{F}_{\tau}\left(3 \frac{A_{b, G} \int u_{b}-\int u_{b} e_{b}}{1 / Y_{b, G}+6 \int u_{b}}+\frac{A_{\nu, G} \int u_{\nu}-\int u_{\nu} e_{\nu}}{1 / Y_{\nu, G}+4 \int u_{\nu}}\right) \\
& +27\left(\frac{A_{b, G} \int u_{b}-\int u_{b} e_{b}}{1 / Y_{b, G}+6 \int u_{b}}\right)^{2}+5\left(\frac{A_{\nu, G} \int u_{\nu}-\int u_{\nu} e_{\nu}}{1 / Y_{\nu, G}+4 \int u_{\nu}}\right)^{2} \\
& +6\left(\frac{A_{b, G} \int u_{b}-\int u_{b} e_{b}}{1 / Y_{b, G}+6 \int u_{b}}\right)\left(\frac{A_{\nu, G} \int u_{\nu}-\int u_{\nu} e_{\nu}}{1 / Y_{\nu, G}+4 \int u_{\nu}}\right) \\
& -3 \frac{\left(\Sigma_{b, G}+A_{b, G}^{2}\right) \int u_{b}-2 A_{b, G} \int u_{b} e_{b}+\int u_{b} \xi_{b}}{1 / Y_{b, G}+6 \int u_{b}} \\
& -\frac{\left(\Sigma_{\nu, G}+A_{\nu, G}^{2}\right) \int u_{\nu}-2 A_{\nu, G} \int u_{\nu} e_{\nu}+\int u_{\nu} \xi_{\nu}}{1 / Y_{\nu, G}+4 \int u_{\nu}}, \\
& \xi_{\nu}=\tilde{E}_{\nu}+2 \tilde{F}_{\nu}\left(3 \frac{A_{t, G} \int u_{t}-\int u_{t} e_{t}}{1 / Y_{t, G}+6 \int u_{t}}+\frac{A_{\tau, G} \int u_{\tau}-\int u_{\tau} e_{\tau}}{1 / Y_{\tau, G}+4 \int u_{\tau}}\right) \\
& +27\left(\frac{A_{t, G} \int u_{t}-\int u_{t} e_{t}}{1 / Y_{t, G}+6 \int u_{t}}\right)^{2}+5\left(\frac{A_{\tau, G} \int u_{\tau}-\int u_{\tau} e_{\tau}}{1 / Y_{\tau, G}+4 \int u_{\tau}}\right)^{2} \\
& +6\left(\frac{A_{t, G} \int u_{t}-\int u_{t} e_{t}}{1 / Y_{t, G}+6 \int u_{t}}\right)\left(\frac{A_{\tau, G} \int u_{\tau}-\int u_{\tau} e_{\tau}}{1 / Y_{\tau, G}+4 \int u_{\tau}}\right) \\
& -3 \frac{\left(\Sigma_{t, G}+A_{t, G}^{2}\right) \int u_{t}-2 A_{t, G} \int u_{t} e_{t}+\int u_{t} \xi_{t}}{1 / Y_{t, G}+6 \int u_{t}}
\end{aligned}
$$




$$
\begin{gathered}
-\frac{\left(\Sigma_{\tau, G}+A_{\tau, G}^{2}\right) \int u_{\tau}-2 A_{\tau, G} \int u_{\tau} e_{\tau}+\int u_{\tau} \xi_{\tau}}{1 / Y_{\tau, G}+4 \int u_{\tau}} . \\
\Sigma_{\nu}=M_{L_{3}}^{2}+M_{\nu_{3, R}}^{2}+M_{H_{2}}^{2} .
\end{gathered}
$$

\section{References}

[1] J. Wess and B. Zumino, Nucl. Phys. B 70 (1974) 39; P. Fayet and S. Ferrara, Phys. Rept. 32 (1977) 249.

[2] H.P. Nilles, Phys. Rept. 110 (1984) 1; H.E. Haber and G.L. Kane, Phys. Rept. 117 (1985) 75.

[3] E. Witten, Nucl. Phys. B 188 (1981) 513.

[4] S. Dimopoulos, S. Raby, and F. Wilczek, Phys. Rev. D 24 (1981) 1681; L.E. Ibáñez and G.G. Ross, Phys. Lett. B 105 (1981) 439; U. Amaldi, W. de Boer, and H. Fürstenau, Phys. Lett. B 260 (1991) 447; P. Langacker and M. Luo, Phys. Rev. D 44 (1991) 817; J. Ellis, S. Kelley, and D.V. Nanopoulos, Phys. Lett. B 260 (1991) 161.

[5] L.E. Ibáñez and G.G. Ross, Phys. Lett. B 110 (1982) 215.

[6] J.R. Ellis, J.S. Hagelin, D.V. Nanopoulos, K.A. Olive and M. Srednicki, Nucl. Phys. B 238 (1984) 453.

[7] D.Z. Freedman, P. van Nieuwenhuizen and S. Ferrara, Phys. Rev. D 13 (1976) 3214; S. Deser and B. Zumino, Phys. Lett. B 62 (1976) 335.

[8] A.H. Chamseddine, R. Arnowitt, and P. Nath, Phys. Rev. Lett. 49 (1982) 970.

[9] M. Dine and A.E. Nelson, Phys. Rev. D 48 (1993) 1277.

[10] G.F. Giudice, M.A. Luty, H. Murayama and R. Rattazzi, JHEP 9812 (1998) 027; L. Randall and R. Sundrum, Nucl. Phys. B 557 (1999) 79.

[11] D.E. Kaplan, G.D. Kribs and M. Schmaltz, Phys. Rev. D 62 (2000) 035010; Z. Chacko, M.A. Luty, A.E. Nelson and E. Ponton, JHEP 0001 (2000) 003.

[12] M. Cvetič, A. Font, L.E. Ibáñez, D. Lüst and F. Quevedo, Nucl. Phys. B 361 (1991) 194; A. Brignole, L.E. Ibáñez and C. Muñoz, Nucl. Phys. B 422 (1994) 125 [Erratumibid. B 436 (1995) 747]; A. Love and P. Stadler, Nucl. Phys. B 515 (1998) 34.

[13] P. Binetruy, M.K. Gaillard and B.D. Nelson, Nucl. Phys. B 604 (2001) 32.

[14] G.A. Blair, W. Porod and P.M. Zerwas, Phys. Rev. D 63 (2001) 017703. 
[15] I. Hinchliffe et al., Phys. Rev. D 55, 5520 (1997); Atlas Collaboration, Technical Design Report 1999, Vol. II, CERN/LHC/99-15, ATLAS TDR 15.

[16] P.M. Zerwas, Proceedings, 1999 Cargèse Institute for High-Energy Physics, hepph/0003221; H. Murayama and M.E. Peskin, Ann. Rev. Nucl. Part. Sci. 46, (1996) 533; E. Accomando et al., ECFA/DESY LC Working Group, Phys. Rep. 299 (1998) 1.

[17] J.A. Aguilar-Saavedra et al. [ECFA/DESY LC Physics Working Group Collaboration], "TESLA Technical Design Report Part III: Physics at an e+e- Linear Collider," hep$\mathrm{ph} / 0106315$.

[18] B.C. Allanach et al., in Proc. of the APS/DPF/DPB Summer Study on the Future of Particle Physics (Snowmass 2001), ed. R. Davidson and C. Quigg, Eur. Phys. J. C 25 (2002) 113.

[19] T.E. Clark, T.K. Kuo and N. Nakagawa, Phys. Lett. B 115 (1982) 26; L.E. Ibáñez, Phys. Lett. B 114 (1982) 243. Z.Y. Zhao, Nucl. Phys. B 212 (1983) 224; C.S. Aulakh and R.N. Mohapatra, Phys. Rev. D 28 (1983) 217.

[20] M. Gell-Mann, P. Ramond and R. Slansky, in "Supergravity", ed. F. van Nieuwenhuizen and D. Freedman, North Holland 1979; T. Yanagida, Proceedings, "Unified Theory and the Baryon Number in the Universe", KEK 1979; R. N. Mohapatra and G. Senjanovic, Phys. Rev. Lett. 44 (1980) 912.

[21] E. Witten, Phys. Lett. B 155 (1985) 151.

[22] E. Witten, Talk at the Workshop on the Future of Particle Physics, Snowmass 2001.

[23] G. L. Kane, Talk at SUSY02, DESY Hamburg; http://www.desy.de/susy02/.

[24] M.M. Nojiri, K. Fujii, and T. Tsukamoto, Phys. Rev. D 54, 6756 (1996).

[25] G.A. Blair and U. Martyn, Proceedings, LC Workshop, Sitges 1999, hep-ph/9910416.

[26] M. Battaglia, "Physics Signatures at CLIC", hep-ph/0103338.

[27] S. Ferrara, L. Girardello and F. Palumbo, Phys. Rev. D 20 (1979) 403.

[28] J. Hisano and H. Murayama, Phys. Rev. D 49 (1994) 1446.

[29] K. Inoue, A. Kakuto, H. Komatsu, and S. Takeshita, Prog. Theor. Phys. 68, 927 (1982); Erratum, ibid. 70, 330 (1983).

[30] S. Martin and M. Vaughn, Phys. Rev. D50, 2282 (1994); Y. Yamada, Phys. Rev. D 50, 3537 (1994); I. Jack, D.R.T. Jones, Phys. Lett. B333 (1994) 372. 
[31] J. Bagger, K. Matchev, D. Pierce, and R. Zhang, Nucl. Phys. B 491 (1997) 3.

[32] S.Y. Choi, A. Djouadi, M. Guchait, J. Kalinowski, H.S. Song and P.M. Zerwas, Eur. Phys. J. C 14 (2000) 535; S.Y. Choi, J. Kalinowski, G. Moortgat-Pick and P.M. Zerwas, Eur. Phys. J. C 22 (2001) 563 [Addendum-ibid. C 23 (2002) 769].

[33] J.L. Feng and T. Moroi, Phys. Rev. D56, 5692 (1997); J.F. Gunion, T. Han, J. Jiang, S. Mrenna and A. Sopczak, in Proc. of the APS/DPF/DPB Summer Study on the Future of Particle Physics (Snowmass 2001), ed. R. Davidson and C. Quigg, hep-ph/0112334.

[34] A. Bartl, K. Hidaka, T. Kernreiter and W. Porod, hep-ph/0207186.

[35] A. Bartl, H. Eberl, S. Kraml, W. Majerotto, W. Porod and A. Sopczak, Z. Phys. C 76 (1997) 549; A. Bartl, H. Eberl, S. Kraml, W. Majerotto and W. Porod, Eur. Phys. J. directC 6 (2000) 1.

[36] G. Degrassi, P. Slavich and F. Zwirner, Nucl. Phys. B 611 (2001) 403; A. Brignole, G. Degrassi, P. Slavich and F. Zwirner, Nucl. Phys. B 631 (2002) 195.

[37] H. Arason et al., Phys. Rev. D46, 3945 (1992).

[38] J.R. Primack, astro-ph/0205391.

[39] A.L. Kagan and M. Neubert, Eur. Phys. J. C 7, 5 (1999); A. Ali and D. London, Phys. Rept. 320 (1999) 79; D.A. Demir, A. Masiero, and O. Vives, Phys. Rev. D 61, 075009 (2000).

[40] M. Drees and K. Hagiwara, Phys. Rev. D 42, 1709 (1990).

[41] T. Ibrahim and P. Nath, Phys. Rev. D 61 (2000) 095008.

[42] P. Gondolo et al., program "DarkSusy", http://www.physto.se/ edsjo/darksusy

[43] J.L. Feng and D.E. Finnell, Phys. Rev. D49, 2369 (1994); A. Bartl, H. Eberl, S. Kraml, W. Majerotto, W. Porod, EPJdirect C 6, 1 (2000).

[44] J.A. Casas, A. Lleyda, and C. Muñoz, Nucl. Phys. B 471, 3 (1996).

[45] S. Weinberg, Phys. Lett. B 91 (1980) 51.

[46] L.J. Hall, Nucl. Phys. B 178 (1981) 75.

[47] G.G. Ross and R.G. Roberts, Nucl. Phys. B 377 (1992) 571.

[48] P. Langacker and N. Polonsky, Phys. Rev. D 47 (1993) 4028. 
[49] M. Bastero-Gil and J. Perez-Mercader, Phys. Lett. B 322 (1994) 355; M. Bastero-Gil and J. Perez-Mercader, Nucl. Phys. B 450 (1995) 21.

[50] A. Dedes, A.B. Lahanas, J. Rizos and K. Tamvakis, Phys. Rev. D 55 (1997) 2955.

[51] H. Murayama and A. Pierce, Phys. Rev. D 65 (2002) 055009.

[52] D.E. Groom et al. [Particle Data Group Collaboration], Eur. Phys. J. C 15 (2000) 1.

[53] K. Mönig, in "Physics and Experiments with Future Linear $e^{+} e^{-}$Colliders", eds. A. Para, H.E. Fisk, Melville 2001, hep-ex/0101005.

[54] J. Erler, S. Heinemeyer, W. Hollik, G. Weiglein and P.M. Zerwas, Phys. Lett. B 486 (2000) 125.

[55] E. Boos, H.U. Martyn, G. Moortgat-Pick, M. Sachwitz, A. Vologdin and P.M. Zerwas, in preparation.

[56] M. Carena et al., Nucl. Phys. B491, 103 (1997); S.A. Abel and B. Allanach, Phys. Lett. B 415, 371 (1997).

[57] W. Buchmüller and D. Wyler, Phys. Lett. B 521 (2001) 291.

[58] N. Polonsky and A. Pomarol, Phys. Rev. Lett. 73 (1994) 2292; N. Polonsky and A. Pomarol, Phys. Rev. D 51 (1995) 6532; Y. Kawamura, H. Murayama and M. Yamaguchi, Phys. Rev. D 51 (1995) 1337.

[59] C.F. Kolda and S.P. Martin, Phys. Rev. D 53 (1996) 3871.

[60] G.F. Giudice and R. Rattazzi, Phys. Rept. 322 (1998) 419.

[61] E. Witten, Talk at SUSY02, DESY Hamburg; http://www.desy.de/susy02/.

[62] S.P. Martin, Phys. Rev. D 55 (1997) 3177; S. Dimopoulos, G.F. Giudice and A. Pomarol, Phys. Lett. B 389 (1996) 37.

[63] J. Bagger, K. Matchev, D. Pierce, and R. Zhang, Phys. Rev. D 55 (1997) 3188.

[64] S. Ambrosanio and G.A. Blair, Eur. Phys. J. C 12 (2000) 287.

[65] B. C. Allanach, D. Grellscheid and F. Quevedo, JHEP 0205 (2002) 048.

[66] C.H. Chen, M. Drees, and J.F. Gunion, Phys. Rev. D 55 (1997) 330; Erratum, ibid. D 60 (1999) 039901.

[67] L.E. Ibáñez, D. Lüst and G.G. Ross, Phys. Lett. B 272 (1991) 251; J.P. Derendinger, S. Ferrara, C. Kounnas and F. Zwirner, Nucl. Phys. B 372 (1992) 145; V.S. Kaplunovsky, Nucl. Phys. B 307 (1988) 145 [Erratum-ibid. B 382 (1992) 436]. 
[68] D. Kazakov and G. Moultaka, Nucl. Phys. B 577 (2000) 121.

[69] L.E. Ibáñez and C. López, Nucl. Phys. B 233 (1984) 511; L.E. Ibáñez and J. Mas, Nucl. Phys. B 286 (1987) 107. 